\title{
Single-dish and aperture synthesis $H$ I observations towards the Draco nebula. \\ I. The data
}

\author{
U. Herbstmeier ${ }^{1,2}$, P.M.W. Kalberla ${ }^{1}$, U. Mebold ${ }^{1}$, H. Weiland ${ }^{1}$, I. Souvatzis ${ }^{1,3}$, A. Wennmacher ${ }^{1,4}$ and \\ J. Schmitz ${ }^{1}$ \\ 1 Radioastronomisches Institut der Universität Bonn, Auf dem Hügel 71, D-53121 Bonn, Germany \\ pkalberl@astro.uni-bonn.de \\ 2 Max-Planck-Institut für Astronomie, Königstuhl 17, D-69117 Heidelberg, Germany \\ uherbst@mpia-hd.mpg.de \\ 3 Institut für Informatik der Universität Bonn, Abteilung V, Römerstr. 117, D-53117 Bonn, Germany \\ 4 Institut für Geophysik und Meteorologie der Universität zu Köln, Albertus-Magnus-Platz, D-50923 Köln, Germany
}

Received August 25; accepted December 14, 1995

\begin{abstract}
We describe 21-cm line observations of the Draco nebula region and present the results as channel maps. These observations have been performed with the Effelsberg 100-m telescope, the Westerbork Synthesis Radiotelescope, and the Very Large Array. The measurements reveal the full extent of the cloud as well as the detailed morphological and kinematical structure of several subareas. A list of continuum point sources found in the aperture synthesis maps is included.
\end{abstract}

Key words: ISM: clouds — ISM: individual objects: Draco clouds — ISM: structure — radio lines: ISM

\section{Introduction}

The faint reflection nebulosities LBN 406, 412,415 (Lynds $1965)$ at $(l, b) \approx\left(91^{\circ}, 38^{\circ}\right)$ were rediscovered in the $21 \mathrm{~cm}$ line as a narrow (velocity dispersion $\sigma \approx 1.5 \mathrm{kms}^{-1}$ ) emission line feature at $v \approx-21 \mathrm{kms}^{-1}$ (in the present paper $v$ denotes velocities with respect to the Local Standard of Rest, LSR) with brightness temperatures up to $20 \mathrm{~K}$ by Goerigk et al. (1983). According to its LSR velocity this cloud can be classified as an intermediate velocity cloud (IVC) and is therefore named IVC G091.0 + 38.0 (VLSR= $-21)$. As it is located in the constellation Draco it is also called Draco nebula or more shortly Dracula.

The results of Goerigk et al. (1983) show that the cloud extends beyond the limits of the field which had been observed at that time. Therefore measurements of the $21 \mathrm{~cm}$ line emission have been obtained for the whole cloud in subsequent years with the Effelsberg $100 \mathrm{~m}$ telescope of the Max-Plank-Institut für Radioastronomie. Additional high resolution $21 \mathrm{~cm}$ line observations of parts of the Draco nebula along the filaments described in Goerigk et al. (1983) have been performed with the Westerbork

Send offprint requests to: U. Herbstmeier, MPIA
Synthesis Radio Telescope (WSRT) ${ }^{1}$ and the Very Large Array (VLA) of the National Radio Astronomy Observatory (NRAO) ${ }^{2}$.

Here we present the complete set of $21 \mathrm{~cm}$ line data. These data are of particular interest because previous studies have shown that the Draco nebula is a remarkable object:

It is the best-studied of the three known highlatitude IVCs showing CO-emission. In addition, the molecules $\mathrm{NH}_{3}$ and $\mathrm{H}_{2} \mathrm{CO}$ have been found (Goerigk et al. 1983; Mebold et al. 1985; Magnani et al. 1985 as MBM 41,42,43,44, Mebold et al. 1987; Lada \& Blitz 1988; Rohlfs et al. 1989; Rohlfs 1989; Mebold et al. 1989; Herbstmeier et al. 1993; Herbstmeier et al. 1994b) and the nebula is seen as an infrared cirrus cloud in the four

\footnotetext{
${ }^{1}$ The Westerbork Synthesis Radio Telescope is operated by the Netherlands Foundation for Radio Astronomy (SZRM) which was financially supported by the Netherlands Organization for the Advancement of Pure Research (ZWO).

${ }^{2}$ The National Radio Astronomy Observatory is operated by Associated Universities, Inc., under cooperative agreement with the National Science Foundation.
} 
bands of the IRAS ${ }^{3}$ survey (Herbstmeier 1984; Odenwald \& Rickard 1987; Odenwald 1988; Heiles et al. 1988 as G90.0 + 38.8 and G94.8+37.6, Herbstmeier et al. 1993). Herbstmeier et al. (1993) have used the H I data in comparison with the IRAS $100 \mu \mathrm{m}$, and $\mathrm{CO}$ observations to derive the molecular hydrogen column densities $N\left(\mathrm{H}_{2}\right)$. They find the lowest values up to now for the ratio of the molecular hydrogen column density and the ${ }^{12} \mathrm{CO}$ $J=1 \rightarrow 0$ line integral: $0.0210^{20}<N\left(\mathrm{H}_{2}\right) / W\left({ }^{12} \mathrm{CO}\right)$ $<0.2610^{20} \mathrm{~cm}^{-2}\left(\mathrm{~K} \mathrm{kms}^{-2}\right)^{-1}$. This indicates a rather large $\mathrm{CO}$ abundance relative to the molecular hydrogen content.

Extinction studies indicate (Goerigk \& Mebold 1986) that the nebula is located more than $500 \mathrm{pc}$ above the galactic plane (see also Mebold et al. 1985; Rohlfs 1986; Wennmacher 1988). Lilienthal et al. (1991) derive a lower distance limit of $300 \mathrm{pc}$ from the lack of interstellar $\mathrm{NaI} \mathrm{D}$ absorption lines. It appears that the Draco nebula is located outside the bulk of the molecular cloud $z$ distribution.

This location made the Draco nebula an ideal target for a search for soft $\mathrm{X}$-ray emission originating outside the galactic disk. In the surroundings of the Draco nebula Hirth et al. (1985) found an enhancement of the soft X-ray $B$ - and $C$-band emission in data observed by McCammon et al. (1983) and Marshall \& Clark (1984). The ROSAT ${ }^{4}$ $1 / 4 \mathrm{keV}$ soft $\mathrm{X}$-ray survey (Trümper 1983) was the basis of the discovery of a detailed anticorrelation between the soft $\mathrm{X}$-ray count rates and the $\mathrm{H}$ I column density distribution of the nebula (Snowden et al. 1991; Burrows \& Mendenhall 1991). This can only be explained by the Draco nebula casting a shadow in the soft $\mathrm{X}$-ray background (SXRB). Thus the nebula became the crucial probe in the discovery of a galactic $\mathrm{X}$-ray corona (Herbstmeier et al. 1994a; Mebold et al. 1993; Hirth et al. 1991; Kerp 1994). Of similar importance is the fact that the depth of the X-ray shadow is a measure of the total column density of absorbing material. Thus, from the difference of that column density and the column densities derived in the present paper one can estimate the molecular hydrogen content independently of the $\mathrm{CO}$ content.

Peculiar features of the Draco nebula, such as its location outside the galactic disk, its large $\mathrm{CO}$ and low $\mathrm{H}_{2}$ content, and a prominent velocity gradient which is observed perpendicular to the boundary facing the galactic centre direction (Goerigk et al. 1983; Herbstmeier 1984; Souvatzis 1989), may be related to a coincidence with HI high velocity clouds (HVCs), (Kalberla et al. 1984;

\footnotetext{
${ }^{3}$ The Infrared Astronomical Satellite, IRAS, was a joint mission of the United States, the Netherlands, and the UK.

${ }^{4}$ The Röntgensatellit ROSAT is a joint mission of Germany, the United States and the UK.
}

Herbstmeier 1984; Mebold et al. 1985; Rohlfs et al. 1989; Verschuur 1990; Mebold et al. 1990).

The data base presented here will allow more detailed studies of these and other peculiar features. We present the $\mathrm{H}$ I data in the form of channel maps, i.e. the distribution of the mean brightness temperature in a given velocity interval. Maps are presented for the range from -210 to $+3 \mathrm{kms}^{-1}$. The observational parameters, the data reduction procedures and the final maps are described in Sect. 2 for the spectra obtained with the $100 \mathrm{~m}$ telescope, and in Sect. 4 for the aperture synthesis maps. The features found in the $21 \mathrm{~cm}$ data will be discussed in more detail in a subsequent paper.

\section{Observations and data reduction of the $H_{I}$ spectra obtained at Effelsberg}

\subsection{The observations}

The observations were made with the $100 \mathrm{~m}$ telescope of the Max-Planck-Institut für Radioastronomie at Effelsberg during several periods in the years 1977, 1978, 1980, 1981-1984, 1988-1990, 1991-1993 (see Table 1). At $21 \mathrm{~cm}$ the beamwidth (HPBW) of the telescope is 9.1' and the main beam efficiency is 0.72 . During the observing period 1977-1980 uncooled, cooled during 1982-1984, dual channel parametric amplifiers with about $80 \mathrm{~K}$ (uncooled) and $45 \mathrm{~K}$ (cooled) cold sky system temperatures and a 384 channel autocorrelation spectrometer were used. In the years 1988-1990 the spectra were obtained with a 1024channel autocorrelation spectrometer and a dual channel cooled FET amplifier which had a cold sky system temperature of $45 \mathrm{~K}$ to $50 \mathrm{~K}$. In 1991 a new cooled dual channel HEMT receiver has been installed with an improved cold sky system temperature between $20 \mathrm{~K}$ and $30 \mathrm{~K}$.

During 1977-1984 and in 1993 the spectrometer was used in the dual channel mode with the spectrometer split into two identical halves. From 1988-1992 all spectra, except few rare cases, were recorded feeding only one frontend channel into the autocorrelation spectrometer. All spectra were observed using a frequency switching technique.

The observation grids were always defined in galactic coordinates $(l, b)$. The grid parameters, however, vary for the different observing periods. In Table 1 we list the main characteristics of the observing grid, the total velocity coverage, $\Delta v$, the velocity resolution, $\delta v$, the integration time, $t_{\text {int }}$, and the $1 \sigma$ rms noise temperature, $\Delta T$, for each spectrometer channel, RX. In Fig. 1 we plot the observing grid in the $(l, b)$ coordinate system distinguishing between the different observing periods listed in Table 1. The observations of period 1977-1980 have been described in full detail by Goerigk et al. (1983). 
Table 1. Parameters of the $21 \mathrm{~cm}$ line observations with the Effelsberg telescope

\begin{tabular}{|c|c|c|c|c|c|c|c|}
\hline \multicolumn{2}{|l|}{ Period } & Grid & $\mathrm{RX}$ & $\begin{array}{r}\Delta v \\
\left(\mathrm{~km} \mathrm{~s}^{-1}\right) \\
\end{array}$ & $\begin{array}{r}\delta v \\
\left(\mathrm{kms}^{-1}\right) \\
\end{array}$ & $\begin{array}{r}t_{\text {int }} \\
(\mathrm{s}) \\
\end{array}$ & $\begin{array}{l}\Delta T \\
(\mathrm{~K}) \\
\end{array}$ \\
\hline \multirow[t]{3}{*}{$\overline{1977-1980}$} & & hexagonal & 1 & $(-313,213)$ & 2.75 & 150 & 0.21 \\
\hline & & $\left(\Delta^{a}=9^{b}\right)$ & & $(-313,263)^{d}$ & & $(78,580)^{d}$ & $(0.1,1.0)^{d}$ \\
\hline & & & 2 & $\begin{array}{c}(-181,81) \\
(-181,131)^{d}\end{array}$ & 1.37 & & 0.39 \\
\hline \multirow{2}{*}{\multicolumn{2}{|c|}{$1982-1983$}} & $\begin{array}{l}\text { orthogonal } \\
\left(10^{\prime}, 10^{\prime}\right)^{b}\end{array}$ & 1 & $\begin{array}{r}(-283,183) \\
(88,264)^{d}\end{array}$ & 2.75 & 176 & 0.14 \\
\hline & & & 2 & $(-151,111)$ & 1.37 & & 0.17 \\
\hline \multirow{2}{*}{\multicolumn{2}{|c|}{1984}} & orthogonal & 1 & $(-363,163)$ & 2.75 & 147 & 0.15 \\
\hline & & $\left(10^{\prime}, 10^{\prime}\right)^{c}$ & 2 & $(-231,31)$ & 1.37 & & 0.35 \\
\hline \multirow[t]{4}{*}{$1988-1989$} & A & $\begin{array}{l}\text { orthogonal } \\
\left(10^{\prime}, 10^{\prime}\right)^{c}\end{array}$ & 1 & $\begin{array}{r}(-214,115) \\
(63,111)^{d}\end{array}$ & 0.32 & 95 & 0.36 \\
\hline & B & & 1 & $(-264,64)$ & 0.32 & 95 & 0.36 \\
\hline & $\mathrm{C}$ & & 1 & $\begin{array}{c}(-329,329) \\
(-429,329)^{d}\end{array}$ & 1.29 & 95 & 0.17 \\
\hline & & & 2 & 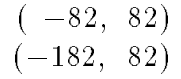 & 0.32 & & 0.36 \\
\hline 1990 & & $\begin{array}{l}\text { orthogonal } \\
\left(10^{\prime}, 10^{\prime}\right)^{c}\end{array}$ & 1 & $(-429,229)$ & 0.64 & 47 & 0.38 \\
\hline \multirow[t]{3}{*}{ 1991-1992 } & A & $\begin{array}{l}\text { orthogonal } \\
\left(10^{\prime}, 10^{\prime}\right)^{c}\end{array}$ & 1 & $(-379,279)$ & 0.64 & 31 & 0.27 \\
\hline & B & & 1 & $(-349,310)$ & 1.29 & 31 & 0.20 \\
\hline & & & 2 & $(-102,63)$ & 0.32 & 31 & 0.41 \\
\hline \multirow[t]{4}{*}{1993} & A & orthogonal & 1 & $(-380,279)$ & 1.29 & 31 & 0.25 \\
\hline & & $\left(10^{\prime}, 10^{\prime}\right)^{c}$ & 2 & $(-380,279)$ & 1.29 & 31 & 0.25 \\
\hline & B & & 1 & $(-380,279)$ & 1.29 & 31 & 0.11 \\
\hline & & & 2 & $(-215,114)$ & 0.64 & 31 & 0.80 \\
\hline
\end{tabular}

${ }^{a}$ : Distance between hexagon points

${ }^{b}$ : Distance in true angles

${ }^{c}$ : Distance in apparent angles

${ }^{d}$ : Maximum range of parameters deviating from the listed typical values.

\subsection{Data reduction and results}

The data processing has been performed using the standard reduction programs of the Max-Planck-Institut für Radioastronomie and the Radioastronomisches Institut der Universität Bonn. The spectra were calibrated according to the IAU standard position $\mathrm{S} 7$ at $(l, b)=$ $\left(132^{\circ},-1^{\circ}\right)$. In the periods $1977-1980$ and $1991-1993$ the standard baseline position $\mathrm{B} 8,(l, b)=\left(90^{\circ}, 40^{\circ}\right)$, was also observed regularly as a stray radiation calibration position. All spectra have been corrected for stray radiation arising from the sidelobes of the telescope and converted to brightness temperature $T_{\mathrm{B}}$ (Kalberla et al. 1980, 1982). From all spectra polynomial baselines with orders up to three, (in a few rare cases up to four) were subtracted. They were derived by fits to velocity ranges with no $21 \mathrm{~cm}$ line emission.

To present the data we have calculated channel maps from the whole set of spectra. As the observing grid is not regular and the velocity resolution is not the same for all spectra we have interpolated the intensity values. This was done in galactic coordinates and in the velocity domain. For each velocity interval (i.e. one channel map) a regular grid in galactic coordinates with a grid spacing of $3^{\prime} \times 3^{\prime}$ was chosen. A gaussian weighting function with $9^{\prime}$ full width at half maximum (FWHM) was applied to interpolate the neighbouring spectra located within a radius of about $3 \times \sigma$ around the grid point. Here $\sigma$ is the dispersion of the gaussian function. The resolution of the final images is therefore $12.7^{\prime}$. Additionally, each spectrum was interpolated also in the velocity range to achieve a regular channel map grid. This was done by taking the weighted average of all velocity channels contributing to the channel map interval. The weight was determined from the fraction of the velocity interval covering the final channel map range. In Fig. 2 the final maps are shown as grey scale plots. The plotted dynamic range (white to black) is given in the header of each image. The low cutoff level corresponds to $3\langle\Delta T\rangle_{\text {m }}$ with $\langle\Delta T\rangle_{\text {m }}$ the mean rms noise temperature of the maps. The widths of the velocity interval as well as the central velocities are indicated in the plot. 


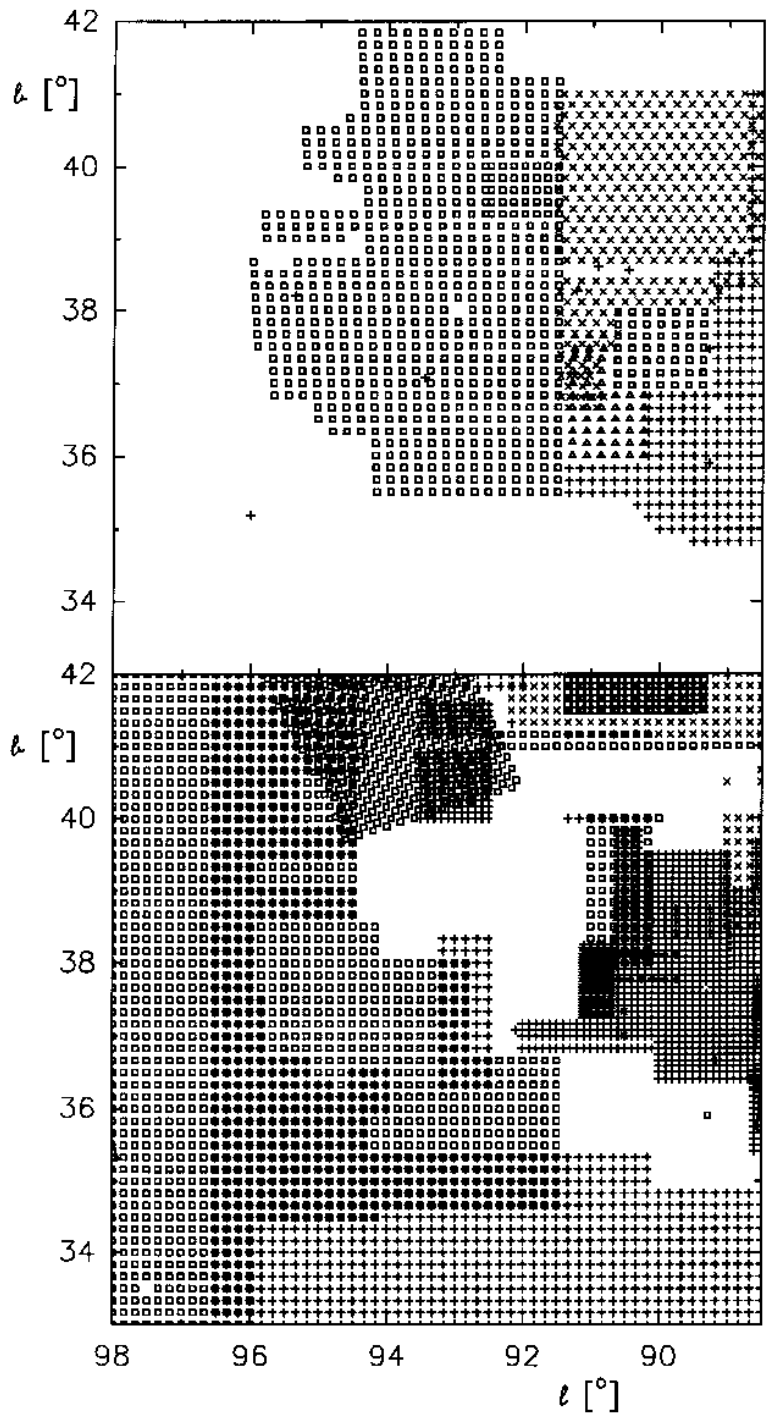

Fig. 1. a) (top) The positions in the galactic coordinate system where H I spectra have been observed at Effelsberg. The observing periods are distinguished by the following symbols: $\times 1977-1980, \square 1982-1983, \triangle 1984,+1988-1989$. b) (bottom) Same as a) for the periods: $\times 1990, \square$ 1991-1992, + 1993

As mentioned in Sect. 2.1 the observations have been collected during different periods. A combination of data with various instrumental setups are suspect of systematic errors. In fact in Fig. 2 rectangular structures are visible at intermediate velocities at longitudes $\approx 96^{\circ}$ to $98^{\circ}$. These are spurious features corresponding to observations in 1991 to 1993. For most of the affected observations multiple observations at several periods exist which were used for consistency checks. In addition we used the repeated observations at the calibration position $\mathrm{B} 8$. Checking for calibration errors no significant deviations within the expected error of $\approx 2 \%$ were found. Comparing spectra at different observing periods we were able to estimate the errors due to the stray radiation corrections. The computer program has been revised making use of a fast 64 bit processor which allowed to improve the accuracy of the calculations considerably. We replaced the Berkeley Survey by the more sensitive Leiden Dwingeloo survey (Hartmann 1994) as the data base from which the stray radiation is calculated. Comparing multiple observations and different program versions we found deviations which are usually less than $0.1 \mathrm{~K}$ and amounted up to $0.2 \mathrm{~K}$ in a few cases only. Such deviations however did not correspond to the velocities at which the stripes are visible. We conclude that the stripes in Fig. 2 are due to low level interference causing systematic baseline ripples which could not be corrected at intermediate velocities. The central parts of the maps in Fig. 2 should be free of systematic effects. Visible features have a signal to noise level better than three. The outer regions are less significant but were included in the plots for sake of completeness.

\section{Some results of the single dish observations}

In the velocity range -210 to $-90 \mathrm{~km} \mathrm{~s}^{-1}$ filamentary and clumpy high-velocity clouds (HVCs) are visible. They belong to the so called HVC Complex $\mathrm{C}$ described e.g. by Wakker (1991). This part of Complex C shows filamentary structure with major axes oriented towards low galactic latitudes and longitudes $\left(\mathrm{GPA} \approx 60^{\circ}\right)$. A velocity gradient is indicated with the fastest clouds lying at the edge towards low latitudes but high longitudes. The velocity range -80 to $-30 \mathrm{~km} \mathrm{~s}^{-1}$ shows very fuzzy so called intermediate velocity clouds (IVCs). They are probably related to the nearby IVC loop GS $155+38-58$ (Heiles 1984). The velocity structure of the Draco nebula filaments can be seen in the interval -30 to $-10 \mathrm{~km} \mathrm{~s}^{-1}$. Velocity gradients are clearly visible which will be discussed elsewhere (see also Goerigk et al. 1983). Towards the low galactic part of our figures, bright low velocity filaments $(v \approx-2$ $\mathrm{km} \mathrm{s}^{-1}$ ) dominate the emission of the diffuse ISM in the velocity range from -10 to $+8 \mathrm{kms}^{-1}$. These filaments are discussed in Wennmacher (1994) and Lilienthal \& Wennmacher (1990).

\section{Observations and data reduction of the $\mathrm{HI}$ spectra observed with aperture synthesis telescopes}

\subsection{Observational parameters}

In 1984 and 1986 two fields in the region of the Draco nebula have been synthesized using the WSRT. The coordinates of the pointing centres in right ascension, $\alpha_{1950}$, and declination, $\delta_{1950}$, as well as in galactic coordinates, $l, b$, are listed in Table 2 (see also Fig. 3). Also listed are the observing dates, the minimum and maximum baselines, $B_{\min }, B_{\max }$, the hour angle interval, $h$, which has been the same for all coverages, the field size in right ascension, 
velocity -207.5 to -182.5 by $5 \mathrm{~km} / \mathrm{s}$

amplitude 0.1 to $2.0 \mathrm{~K}$

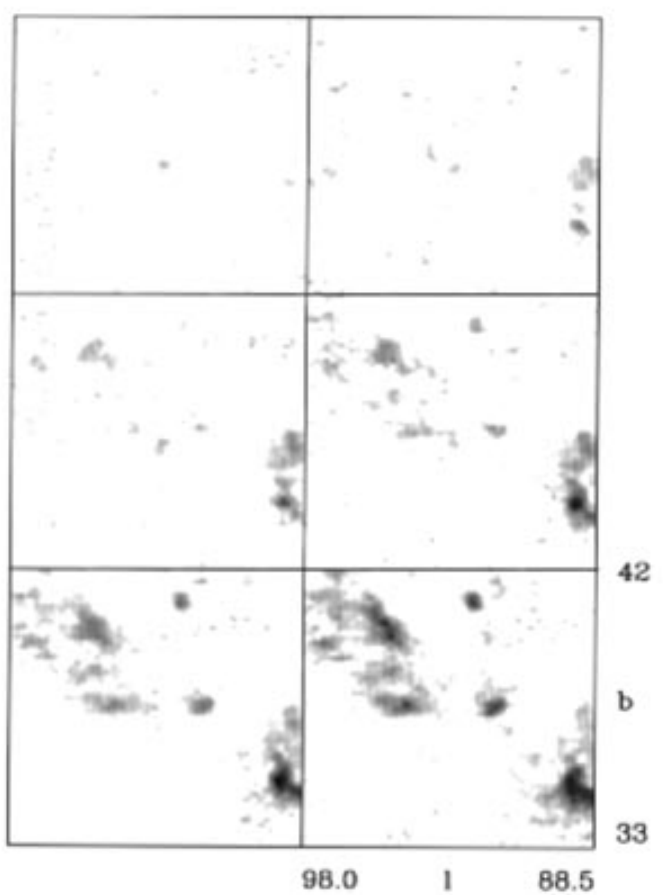

velocity -147.5 to -122.5 by $5 \mathrm{~km} / \mathrm{s}$

amplitude 0.1 to $2.0 \mathrm{~K}$

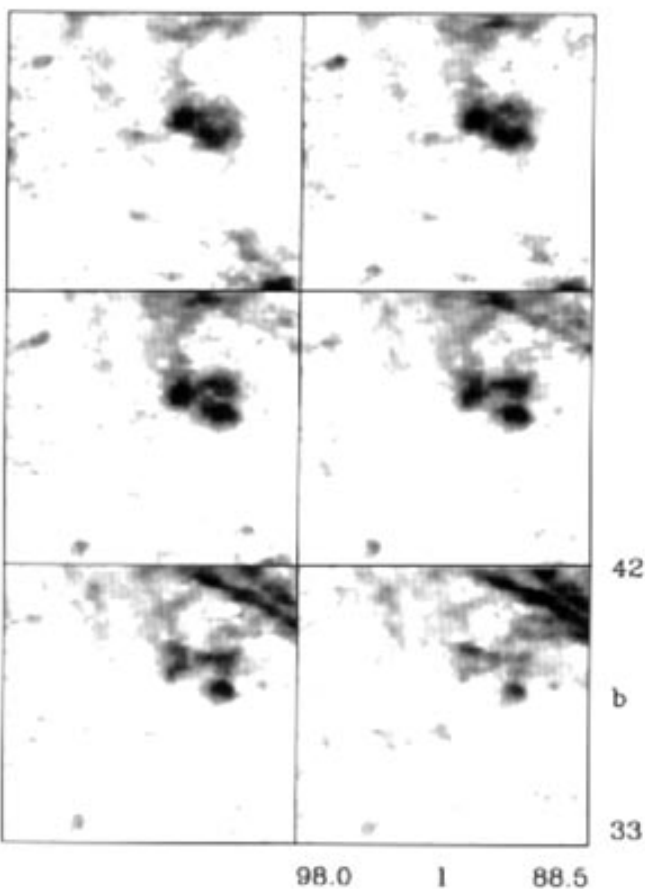

velocity -177.5 to -152.5 by $5 \mathrm{~km} / \mathrm{s}$

amplitude 0.1 to $2.0 \mathrm{~K}$

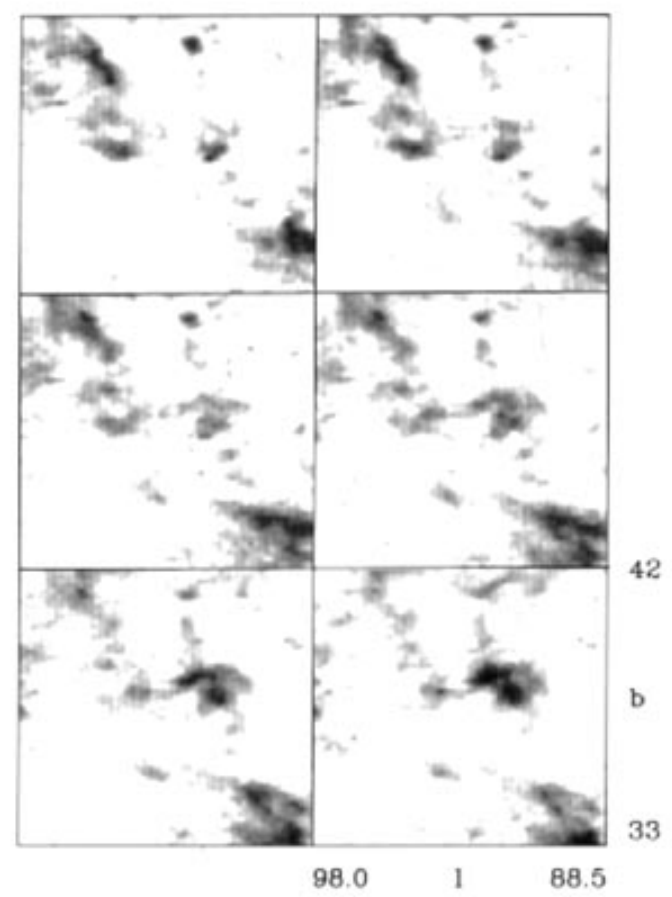

velocity -117.5 to -92.5 by $5 \mathrm{~km} / \mathrm{s}$

amplitude 0.1 to $2.0 \mathrm{~K}$

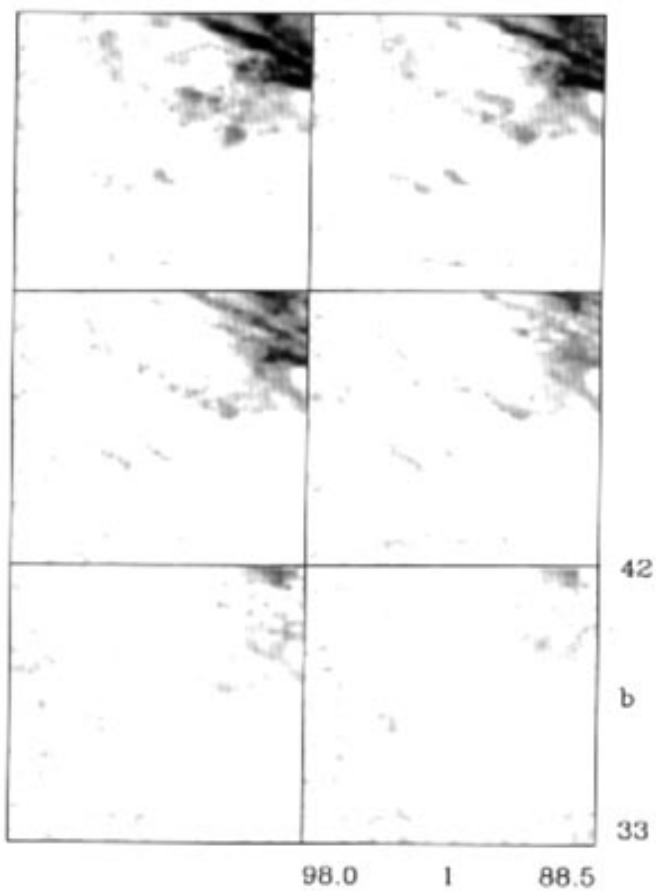

Fig. 2. The H I channel maps of the mean brightness temperature for the Effelsberg $21 \mathrm{~cm}$ spectra. The central velocities and the widths of the velocity intervals are indicated as well as the grey scale range (white to black) 
velocity -87.5 to -62.5 by $5 \mathrm{~km} / \mathrm{s}$

amplitude 0.1 to $2.0 \mathrm{~K}$

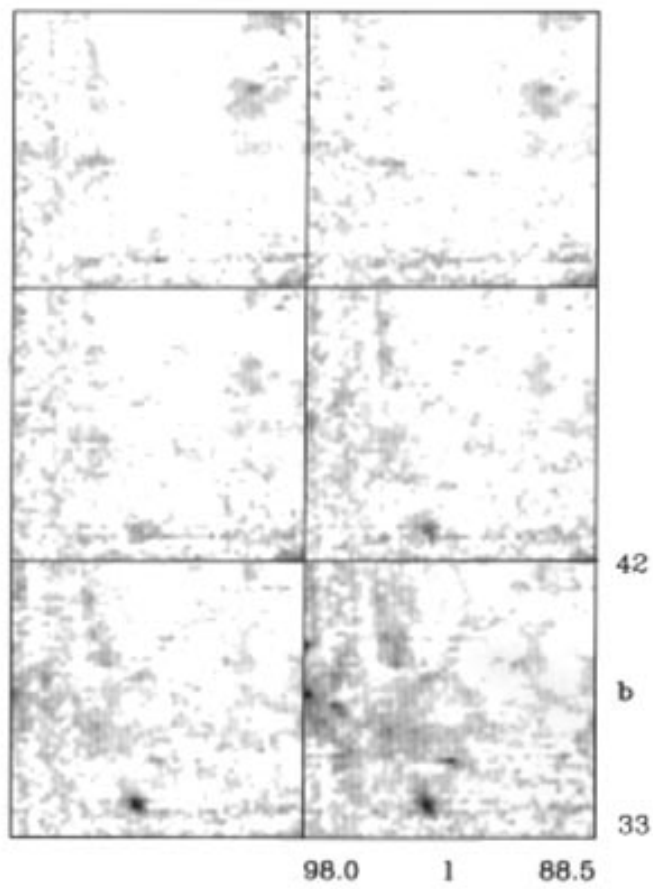

velocity -50.25 to -42.75 by $1.5 \mathrm{~km} / \mathrm{s}$ amplitude 0.2 to $3.0 \mathrm{~K}$

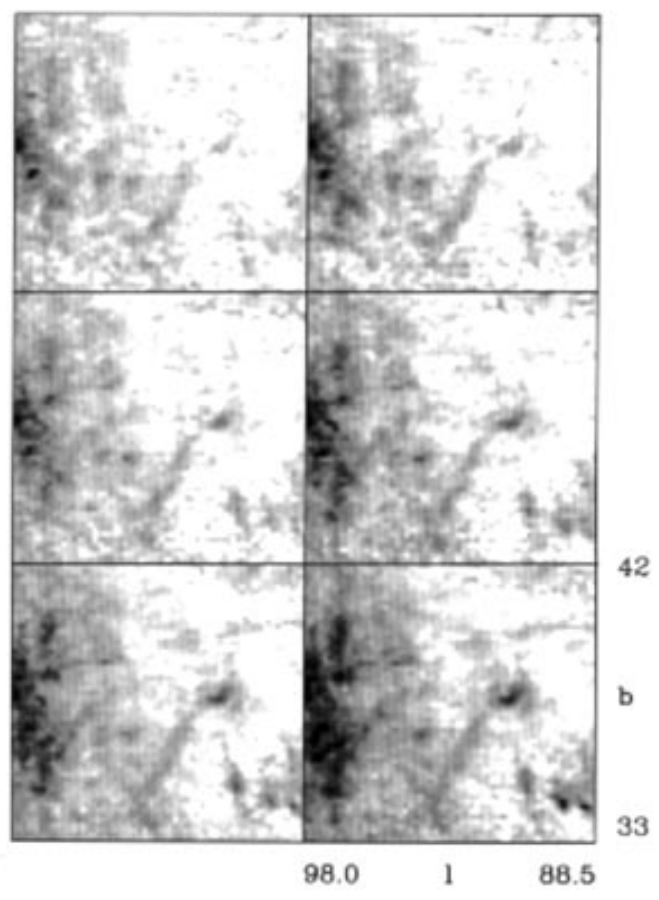

velocity -59.25 to -51.75 by $1.5 \mathrm{~km} / \mathrm{s}$ amplitude 0.2 to $2.0 \mathrm{~K}$

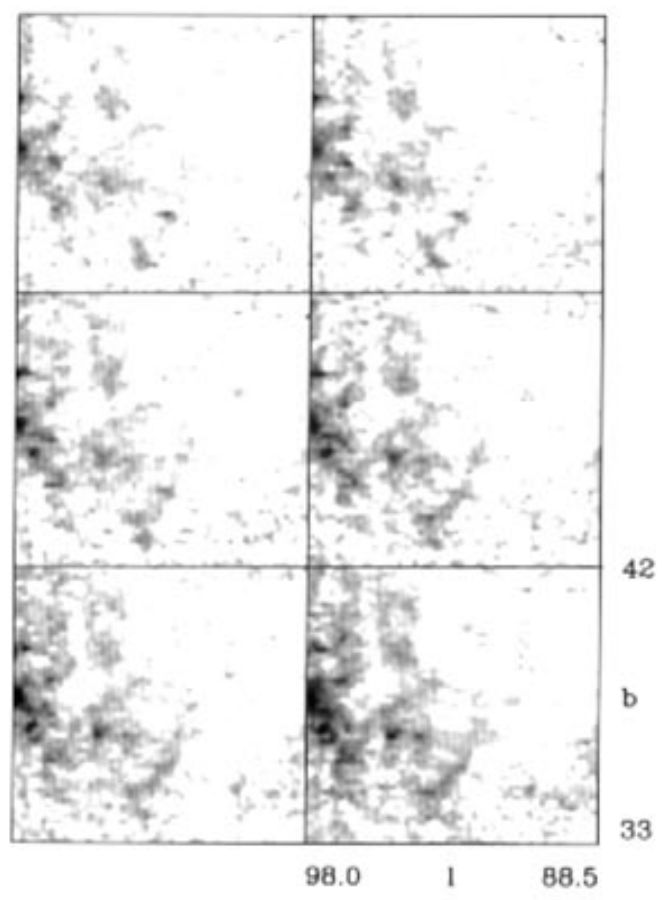

velocity -41.25 to -33.75 by $1.5 \mathrm{~km} / \mathrm{s}$

amplitude 0.2 to $4.0 \mathrm{~K}$

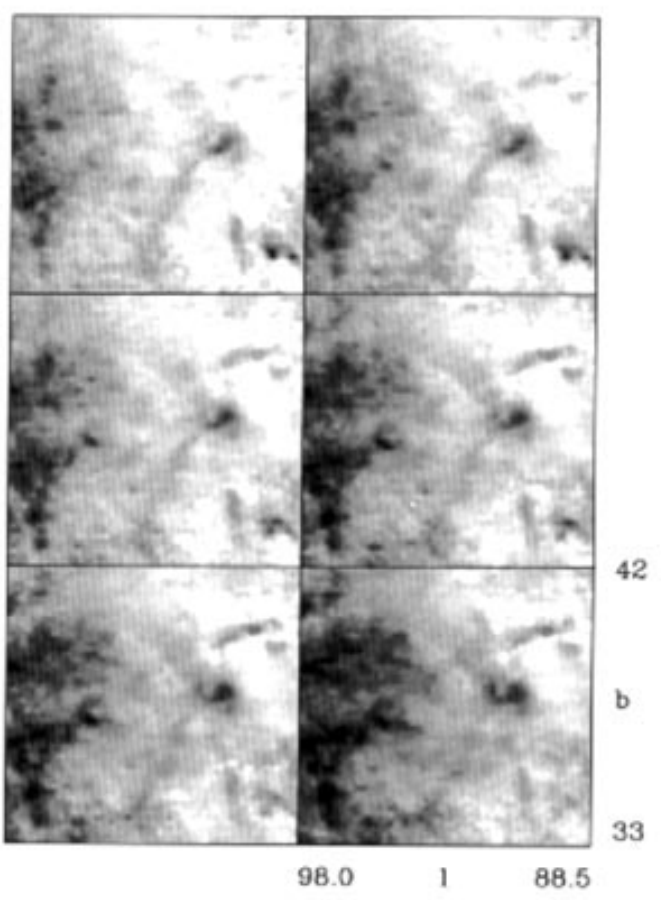

Fig. 2. continued 
velocity -32.25 to -24.75 by $1.5 \mathrm{~km} / \mathrm{s}$ amplitude 0.2 to $8.0 \mathrm{~K}$

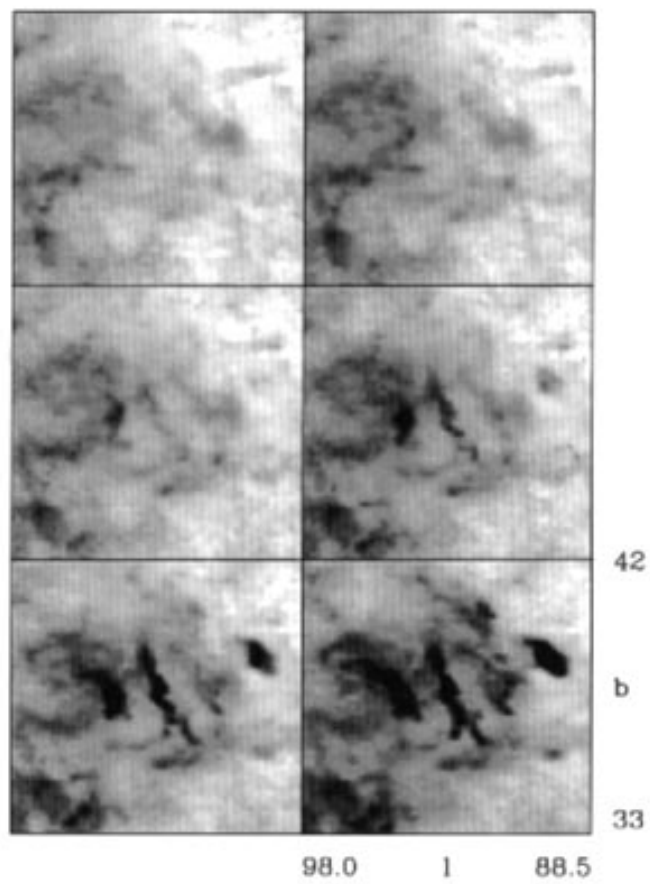

velocity -14.25 to -6.75 by $1.5 \mathrm{~km} / \mathrm{s}$ amplitude 0.2 to $8.0 \mathrm{~K}$

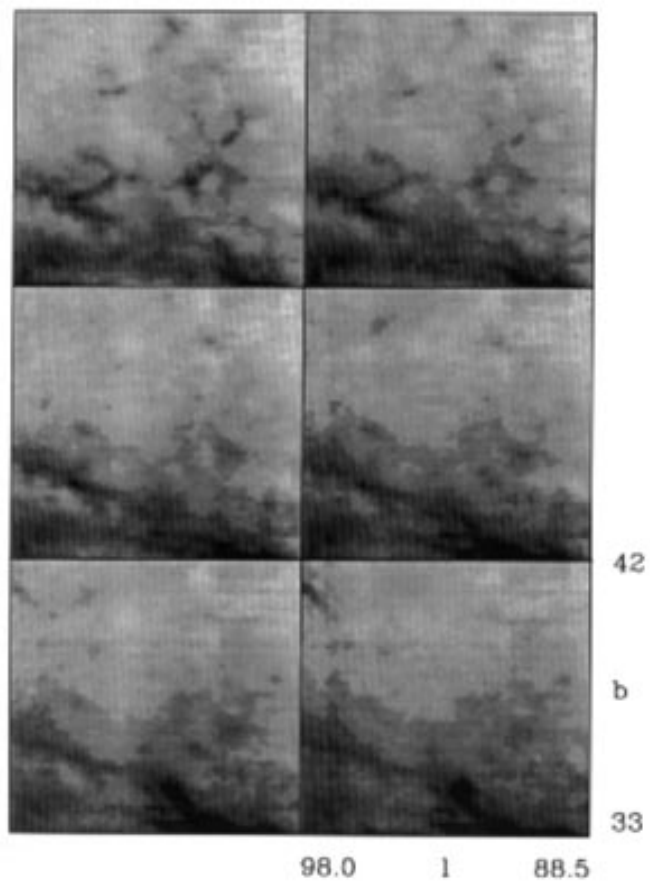

velocity -23.25 to -15.75 by $1.5 \mathrm{~km} / \mathrm{s}$ amplitude 0.2 to $12.0 \mathrm{~K}$

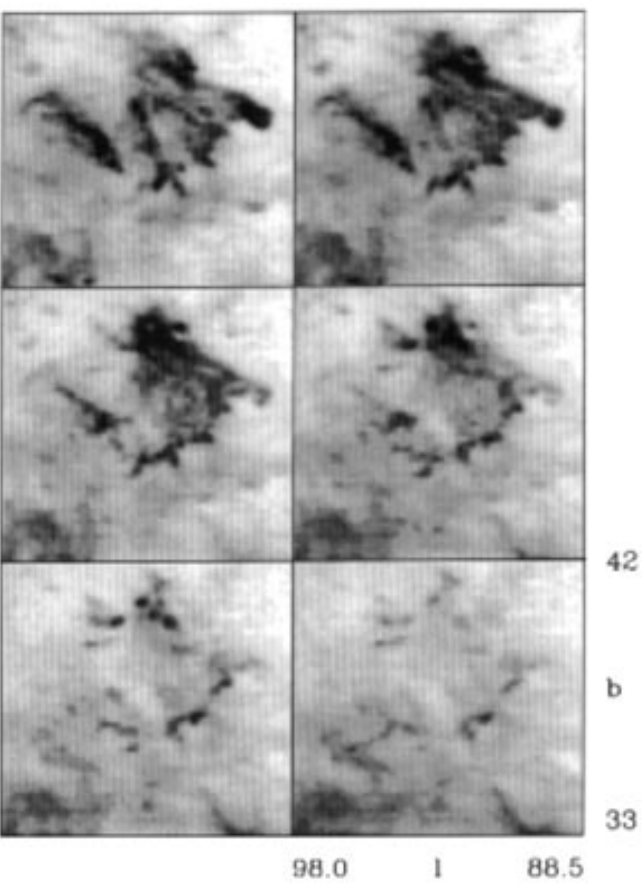

velocity -5.25 to 2.25 by $1.5 \mathrm{~km} / \mathrm{s}$ amplitude 0.2 to $8.0 \mathrm{~K}$

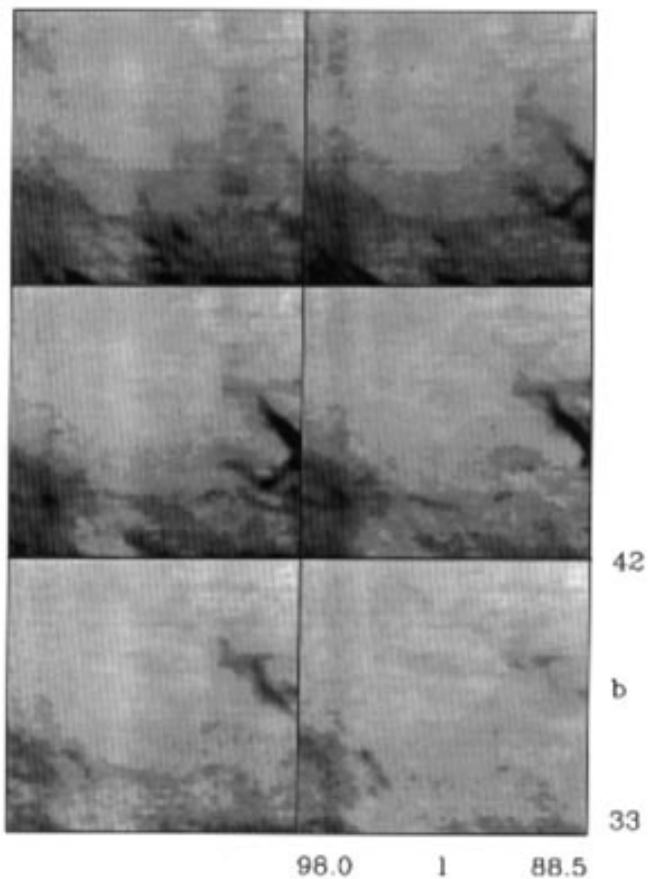

Fig. 2. continued 

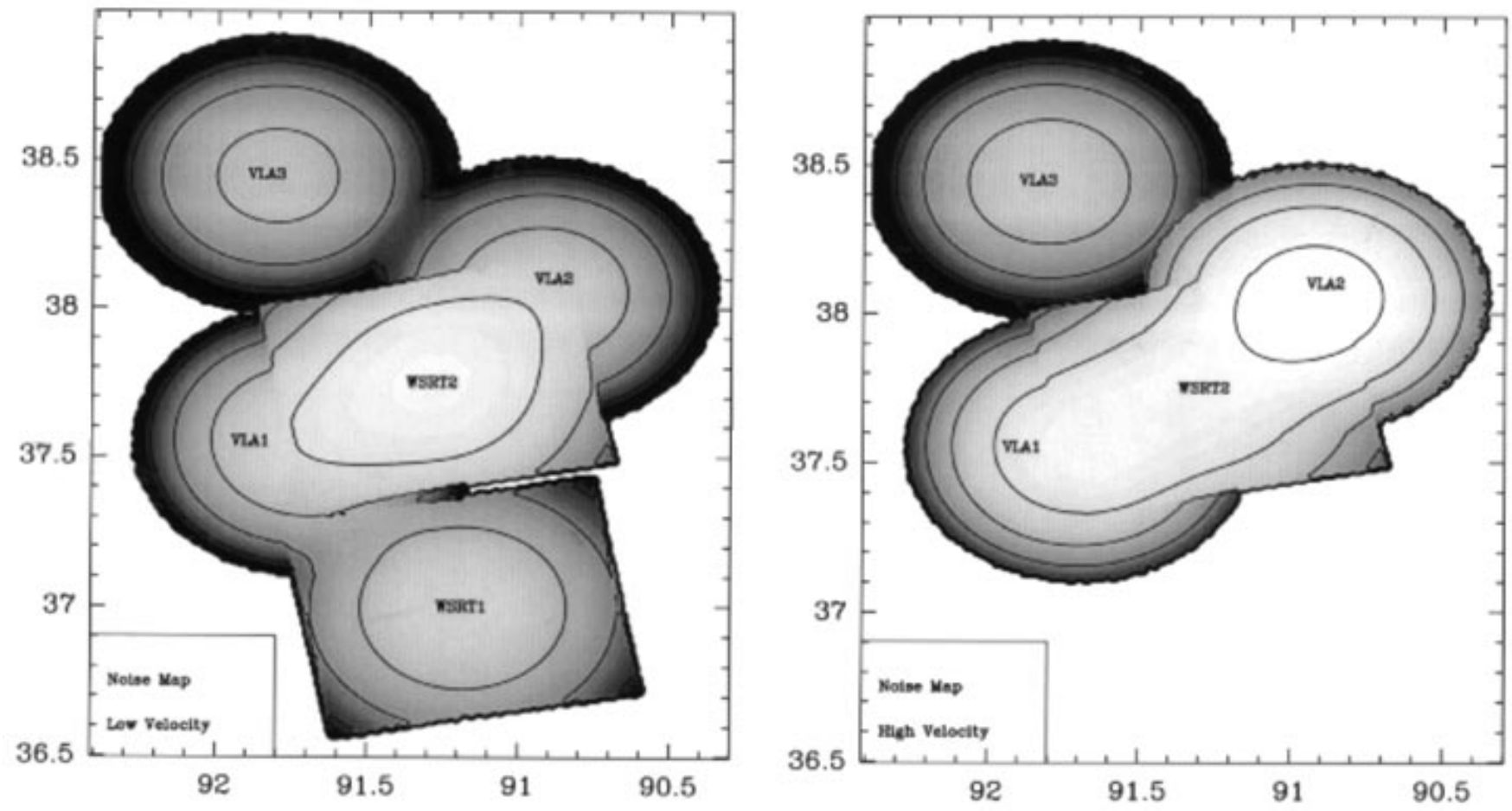

Fig. 3. Noise amplitude of the combined map for five synthesized fields at the edge of the Draco nebula a) for the velocity range -50 to $10 \mathrm{~km} \mathrm{~s}^{-1}$, b) for the velocity range -200 to $-80 \mathrm{~km} \mathrm{~s}^{-1}$ ). The names of the single fields are indicated. The grey scale range from white to black corresponds 0.1 to $3 \mathrm{~K}$. The contours are $0.125,0.25,0.5,1$ and $2 \mathrm{~K}$. From bottom to top are WSRT1, VLA1, WSRT2, VLA2 and VLA3 (see Tables 2 to 4)

$\Delta \alpha$, and declination, $\Delta \delta$, the grid in right ascension, $\mathrm{d} \alpha$, and declination, $\mathrm{d} \delta$, the half power beam width of the synthesized beam in right ascension and declination, HPBW, the velocity coverage, $\Delta v$, the velocity resolution, $\delta v$, and the references where detailed descriptions can be found.

During the WSRT observations telescopes 0-9, A, and B were correlated. In 1983 the data were collected with the 5120-channel correlator of the WSRT (Bos et al. 1981). In 1986 the new 20480-channel correlator was used. All observations were done with 2 polarizations and in the 2-bit correlator mode. An uniform frequency taper and a gaussian taper in the $u v$-plane have been applied. The $u v$-data were calibrated with respect to $3 \mathrm{C} 48$ and $3 \mathrm{C} 147$. During the coverage of the WSRT 2 field with a minimum baseline of $72 \mathrm{~m}$ the antenna 7 was out of order resulting in two ellipses in the $u v$-plane where no data were recorded.

The data were fourier-transformed into channel maps using the standard WSRT packages (Högbom \& Brouw 1974). After this transformation two channel maps of the 1984 observations at $v=-37 \mathrm{kms}^{-1}$ and $v=$ $-34.9 \mathrm{~km} \mathrm{~s}^{-1}$ show strong defects. They have been replaced by the mean values of the two neighbouring channels. All further analyses were done using the Groningen Image Processing System (GIPSY, Allen et al. 1985) complemented by tasks created at the Radioastronomisches Institut der Universität Bonn.
In 1984 and 1985 four additional fields were synthesized with the VLA in its D-configuration. One of these fields (VLA 2) was synthesized in 1984 and again in 1985 with a finer velocity resolution. The coordinates, the telescope configurations, and the spectral parameters are listed in Tables 3 and 4 . To cover both the high velocity $(\mathrm{HV})$ as well as the low velocity range (LV) the central frequency of each observation was switched periodically during one synthesis. This procedure results in almost identical shapes of the synthesized beams. The velocity coverage and the integration time, $t_{\text {int }}$, are therefore listed separately in Tables 3 and 4 . The observations have been performed with a three level quantisation of the 11232-channel correlator. The frequency domain was hanning smoothed, the $u v$-data were gaussian tapered. The $u v$-data were calibrated with respect to $3 \mathrm{C} 286$ and 3C 343. The data were corrected and fourier-transformed to channel maps using the standard reduction facilities of the VLA.

\subsection{Continuum emission and grating rings}

To get the pure H I line signal one has to subtract the emission of continuum sources from all channel maps. Here, we have selected channels which are apparently free of line emission on both ends of the velocity coverage. Channel 
Table 2. The observational parameters of the Westerbork measurements

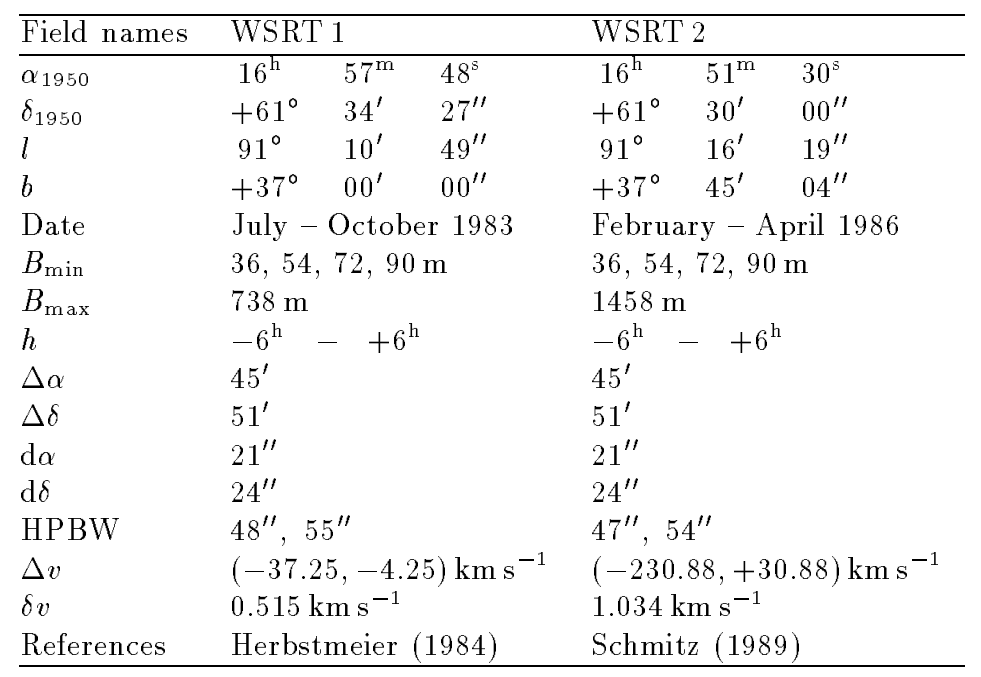

Table 3. The observational parameters of the VLA measurements in autumn 1984

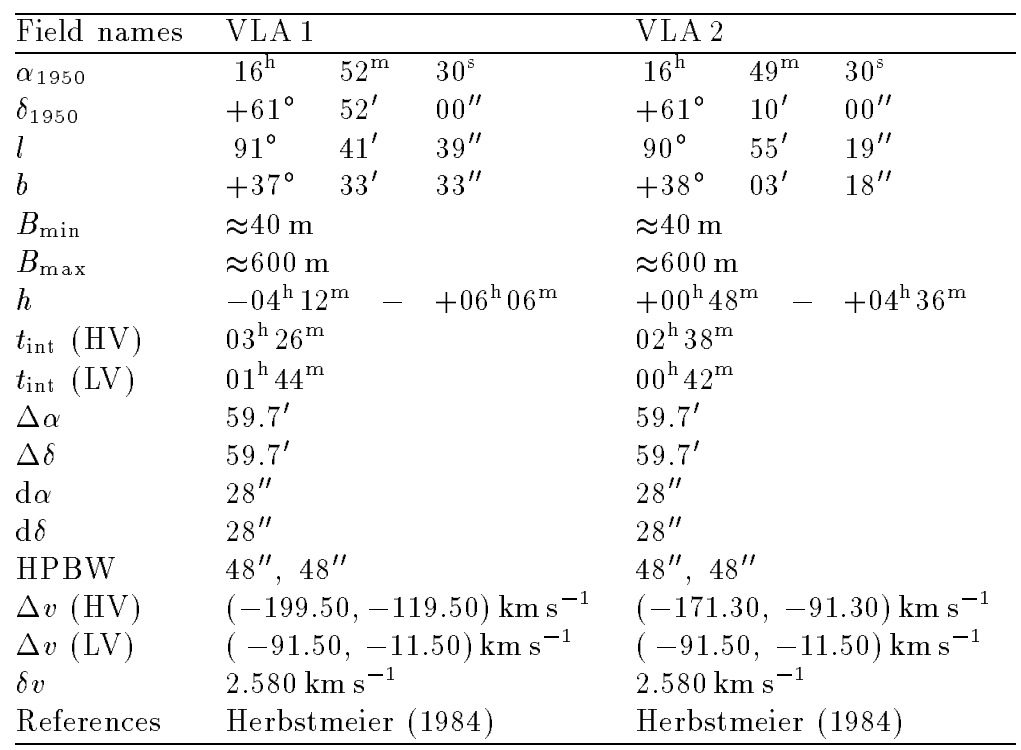

maps with increased noise due to bandpass effects were excluded. The average of these selected maps represents the continuum emission as well as the associated grating rings. Table 5 lists the continuum point sources brighter than $10 \mathrm{mJy}$ found in the fields of view. The uncertainties of the derived source posistions are $3^{\prime \prime}$ to $45^{\prime \prime}\left(1^{\prime \prime}\right.$ to $\left.10^{\prime \prime}\right)$ in right ascension (declination), with most of the sources showing a positional error ellipse of $12^{\prime \prime} \times 3^{\prime \prime}$. The uncertainties in the flux values are in the range of 1 to $5 \mathrm{mJy}$. More details and a search for absorption lines will be discussed elsewhere. The continuum maps have been subtracted from all corresponding line channel maps. This procedure has removed the continuum point sources and their associated grating rings from the channel maps. In general, the amplitude of the convolution of the grating rings is negligible (but see Sect. 4.5). After the full synthesis the radius of the first grating ring of the WSRT 1 observation is $40^{\prime}$ and the maximum amplitude is about $5 \%$ of the main beam. The missing ellipses in the $u v$-plane of the WSRT 2 observation (see Sect. 4.1) lead to periodic grating rings with a period of 2 ' superposed on a beat with a period of $10^{\prime}$. The maximum amplitude of the grating rings has been the same as for the WSRT 1 measurements. The first sidelobes of the different VLA patterns show a maximum of about $7 \%$ of the main beam amplitude. 
Table 4. The observational parameters of the VLA measurements in November 1985

\begin{tabular}{|c|c|c|c|c|c|c|c|}
\hline \multirow{2}{*}{$\frac{\text { Field names }}{\alpha_{1950}}$} & \multicolumn{2}{|l|}{ VLA 2} & \multicolumn{2}{|l|}{ VLA 3} & \multicolumn{3}{|c|}{ VLA 4} \\
\hline & $49^{\mathrm{m}}$ & $30^{5}$ & $45^{\mathrm{m}}$ & $00^{3}$ & $16^{\mathrm{h}}$ & $47^{\mathrm{m}}$ & $00^{5}$ \\
\hline$\delta_{1950}$ & $+61^{\circ}$ & $00^{\prime \prime}$ & $+61^{\circ}$ & $00^{\prime \prime}$ & $+64^{\circ}$ & $10^{\prime}$ & $00^{\prime \prime}$ \\
\hline$l$ & $55^{\prime}$ & $19^{\prime \prime}$ & $47^{\prime}$ & $56^{\prime \prime}$ & $94^{\circ}$ & $42^{\prime}$ & $00^{\prime \prime}$ \\
\hline$b$ & $+38^{\circ} \quad 03^{\prime}$ & $18^{\prime \prime}$ & $+38^{\circ} \quad 26^{\prime}$ & $56^{\prime \prime}$ & $+37^{\circ}$ & $37^{\prime}$ & $48^{\prime \prime}$ \\
\hline$B_{\min }$ & $\approx 40 \mathrm{~m}$ & & $\approx 40 \mathrm{~m}$ & & $\approx 40 \mathrm{l}$ & & \\
\hline$B_{\max }$ & $\approx 600 \mathrm{~m}$ & & $\approx 600 \mathrm{~m}$ & & $\approx 600$ & & \\
\hline$h$ & $-05^{\mathrm{h}} 27^{\mathrm{m}}-$ & $+02^{\mathrm{h}} 35^{\mathrm{m}}$ & $+02^{\mathrm{h}} 30^{\mathrm{m}}-$ & $+07^{\mathrm{h}} 15^{\mathrm{m}}$ & $-06^{\mathrm{h}}$ & $\mathrm{m}$ & $+00^{\mathrm{h}} 38^{\mathrm{m}}$ \\
\hline$t_{\text {int }}(\mathrm{HV})$ & $05^{\mathrm{h}} 10^{\mathrm{m}}$ & & $03^{\mathrm{h}} 40^{\mathrm{m}}$ & & $04^{\mathrm{h}} 4$ & & \\
\hline$t_{\text {int }}(\mathrm{LV})$ & $01^{\mathrm{h}} 20^{\mathrm{m}}$ & & $00^{\mathrm{h}} 50^{\mathrm{m}}$ & & $01^{\mathrm{h}} 1$ & & \\
\hline$\Delta \alpha$ & $59.7^{\prime}$ & & $59.7^{\prime}$ & & $59.7^{\prime}$ & & \\
\hline$\Delta \delta$ & $59.7^{\prime}$ & & $59.7^{\prime}$ & & $59.7^{\prime}$ & & \\
\hline $\mathrm{d} \alpha$ & $28^{\prime \prime}$ & & $28^{\prime \prime}$ & & $28^{\prime \prime}$ & & \\
\hline $\mathrm{d} \delta$ & $28^{\prime \prime}$ & & $28^{\prime \prime}$ & & $28^{\prime \prime}$ & & \\
\hline HPBW (HV) & $46^{\prime \prime}, 45^{\prime \prime}$ & & $59^{\prime \prime}, 43^{\prime \prime}$ & & $48^{\prime \prime}$ & & \\
\hline HPBW (LV) & $51^{\prime \prime}, 50^{\prime \prime}$ & & $69^{\prime \prime}, 47^{\prime \prime}$ & & $55^{\prime \prime}$ & & \\
\hline$\Delta v(\mathrm{HV})$ & $(-148.96,-1$ & $0.31) \mathrm{km} \mathrm{s}^{-1}$ & $(-169.56,-9$ & $2.27) \mathrm{km} \mathrm{s}^{-1}$ & $(-20$ & $21,-1$ & $0.92) \mathrm{km} \mathrm{s}^{-1}$ \\
\hline$\Delta v(\mathrm{LV})$ & $(-39.45,-0$ & 80) $\mathrm{kms}^{-1}$ & $(-76.81,+0$ & 48) $\mathrm{km} \mathrm{s}^{-1}$ & $(-6)$ & $8,+8$ & 21) $\mathrm{km} \mathrm{s}^{-1}$ \\
\hline & $1.288 \mathrm{~km} \mathrm{~s}^{-1}$ & & $2.576 \mathrm{~km} \mathrm{~s}^{-1}$ & & 2.576 & $\mathrm{~ms}^{-1}$ & \\
\hline References & Souvatzis (19 & & Schmitz (198 & & Souv & zis $(19$ & \\
\hline
\end{tabular}

Table 5. Position and flux $S$ of the continuum point sources found in the interferometer fields

\begin{tabular}{rrrrrrrrrr}
\hline No. & $\begin{array}{r}l \\
\left(^{\circ}\right)\end{array}$ & $\begin{array}{r}b \\
\left(^{\circ}\right)\end{array}$ & $\begin{array}{r}\alpha_{1950} \\
(\mathrm{~m})\end{array}$ & $(\mathrm{s})$ & $\left(^{\circ}\right)$ & $\begin{array}{r}\delta_{1950} \\
\left(^{\prime}\right)\end{array}$ & $\left(^{\prime \prime}\right)$ & $\begin{array}{r}S \\
(\mathrm{~m} \mathrm{Jy})\end{array}$ \\
\hline 1 & 90.810 & 37.540 & 16 & 53 & 50 & 61 & 11 & 01 & 34 \\
2 & 90.862 & 38.207 & 16 & 48 & 21 & 61 & 05 & 19 & 143 \\
3 & 90.950 & 38.010 & 16 & 49 & 50 & 61 & 11 & 52 & 19 \\
4 & 91.239 & 36.682 & 17 & 00 & 22 & 61 & 40 & 36 & 290 \\
5 & 91.240 & 37.964 & 16 & 49 & 48 & 61 & 25 & 52 & 205 \\
6 & 91.260 & 37.640 & 16 & 52 & 26 & 61 & 30 & 49 & 13 \\
7 & 91.360 & 38.050 & 16 & 48 & 56 & 61 & 30 & 17 & 12 \\
8 & 91.428 & 37.457 & 16 & 53 & 43 & 61 & 40 & 52 & 16 \\
9 & 91.433 & 37.610 & 16 & 39 & 13 & 61 & 39 & 13 & 42 \\
10 & 91.489 & 37.263 & 16 & 55 & 14 & 61 & 46 & 00 & 57 \\
11 & 91.570 & 37.277 & 16 & 55 & 01 & 61 & 49 & 37 & 59 \\
12 & 91.640 & 37.950 & 16 & 49 & 21 & 61 & 44 & 30 & 18 \\
13 & 91.702 & 37.560 & 16 & 52 & 29 & 61 & 52 & 21 & 13 \\
14 & 91.731 & 38.628 & 16 & 43 & 38 & 61 & 39 & 22 & 90 \\
15 & 91.757 & 37.252 & 16 & 54 & 59 & 61 & 58 & 40 & 18 \\
16 & 91.773 & 38.727 & 16 & 42 & 46 & 61 & 39 & 48 & 76 \\
17 & 91.809 & 37.756 & 16 & 50 & 22 & 61 & 54 & 19 & 11 \\
18 & 91.864 & 37.432 & 16 & 53 & 19 & 62 & 01 & 30 & 14 \\
19 & 91.907 & 37.532 & 16 & 52 & 26 & 62 & 02 & 16 & 24 \\
20 & 91.931 & 38.448 & 16 & 44 & 48 & 61 & 51 & 04 & 29 \\
21 & 94.493 & 37.558 & 16 & 48 & 01 & 64 & 01 & 35 & 13 \\
22 & 94.570 & 37.399 & 16 & 49 & 18 & 64 & 07 & 32 & 23 \\
23 & 94.599 & 37.376 & 16 & 49 & 27 & 64 & 09 & 14 & 11 \\
24 & 94.643 & 37.365 & 16 & 49 & 28 & 64 & 11 & 23 & 15 \\
25 & 94.824 & 37.681 & 16 & 46 & 19 & 64 & 14 & 52 & 16 \\
26 & 94.876 & 37.462 & 16 & 48 & 10 & 64 & 20 & 41 & 23 \\
27 & 94.925 & 37.772 & 16 & 45 & 18 & 64 & 18 & 03 & 13 \\
\hline & & & & & & & & &
\end{tabular}




\subsection{The short spacing and primary beam corrections}

The size of the single telescopes of an interferometer limits the shortest baselines, which can be achieved (see Tables 2-4). The visibilities of these shortest spacings correspond to the emission of extended structures of the H I gas. These structures are not visible for an interferometer. As a result, the synthesized pattern shows negative side lobes and "negative bowls" around emission regions are visible. As the galactic H I emission structure is known to be complex the ususal deconvolution procedures cannot be used to restore the true brightness distribution unequivocally. The best method to fill-in the missing short spacing information is to use the data from large single-dish telscopes (short spacing correction, Bajaja \& van Albada 1979).

On this behalf we have observed all synthesized fields with the $100 \mathrm{~m}$ telescope at Effelsberg. To get almost full sampling, the grid has been chosen to be $5^{\prime}$ in equatorial coordinates for all fields. Table 6 lists the observational parameters of these short spacing observations. We give fieldname (Col. 1), date of observation (Col. 2), number of frontend channels ( $\mathrm{Col} .3$ ), velocity coverage (Col. 4), velocity resolution ( $\mathrm{Col} .5)$, and integration time per position (Col.6). The method to correct the interferometer data is briefly described in the following paragraphs.

First, we have to regrid the Effelsberg data to the $\left(\alpha_{1950}, \delta_{1950}, v\right)$ grid of the interferometer data. This was done for the sky coordinates by convolving the data with a gaussian function of $9^{\prime} \mathrm{HPBW}$ and interpolating to the grid defined by the aperture synthesis observations. The velocity grid was adjusted by a weighted average of the Effelsberg frequency channels. Then we have deconvolved the Effelsberg data to correct for the interpolation. The single-dish main beam has been approximated by a gaussian. Subsequently the primary beam attenuation of the interferometer was applied to the single dish channel maps. The next step was to transform the flux density scale, $S$, of the interferometer data to brightness temperatures, $T_{\mathrm{B}}$, according to

$$
S=2 T_{\mathrm{B}} k_{\mathrm{B}} \lambda^{-2} \int_{\Omega_{\mathrm{MB}}} A(\Omega) \mathrm{d} \Omega
$$

were $\Omega_{\mathrm{MB}}$ is the main beam area, $A(\Omega)$ the antenna pattern, $k_{\mathrm{B}}$ the Boltzmann constant and $\lambda$ the wavelength of the observation. The main beam solid angle has been computed from the synthesized beam pattern adding main beam contributions within the first negative values. The resulting main beam solid angles, $\mathrm{MB}$, and the conversion factors from the flux to the brightness temperature scale, $T_{\mathrm{B}} S^{-1}$ are listed in Table 7 .

Subsequently, the short spacing data have been combined with the interferometer data in the $u v$-plane. The resulting rms-noise brightness temperature $\Delta T$ is listed in Table 7 together with the velocity resolution $\delta v$. For all data sets a good agreement of the interferometer and single dish data in the overlapping range of spatial frequencies has been found. As the final step, the data have been corrected for the primary beam attenuation, i.e. they were divided by the main beam pattern of the interferometer to get the true brightness temperature for the whole field of view. This correction results in an artifical increase of the noise values at the edges of the fields.

\subsection{Combination of the interferometer data sets}

\subsubsection{Merging the data sets of the VLA 2 field}

Souvatzis (1989) has combined the two different data sets of the VLA 2 field. The aim of this combination was:

1. to maintain the highest possible velocity resolution.

2. to increase the signal-to-noise ratio, and

3 . to correct for interference stripes which were visible in some of the channel maps observed in 1985.

To achieve these aims the two data sets were averaged by applying weights which are proportional to the square root of the velocity widths and inversely proportional to the square of the noise of the two sets. The weights, the final velocity resolution, and the noise levels are listed in Table 8 for various velocity intervals of the final data set. For more details see Souvatzis (1989).

4.4.2. Combining the fields along the western edge of the Draco nebula

The five fields WSRT 1, WSRT 2, VLA 1, VLA 2, and VLA 3 are covering a contiguous area of the Draco nebula, the edge facing the galactic center, i.e. the edge towards low latitudes and low longitudes. As they are partly overlapping we have combined these fields. First, the maps of each field have been interpolated to a common velocity grid, and interpolated and transformed to a grid of $28^{\prime \prime}$ spacing in galactic coordinates. Then, for each velocity interval the overlapping parts of the maps have been averaged together using weights which take into account that 1) the noise is different for each of the synthesized primary beam areas and that 2) the noise increases from the centre to the rim within each of these areas. The weights, $g$, are given by

$$
g_{\mathrm{i}}(l, b)=\left(P_{\mathrm{i}}(l, b) \Delta T_{\mathrm{i}}^{-1}\right)^{2}
$$

Here, $P_{\mathrm{i}}(l, b)$ is the primary beam pattern and $\Delta T$ the noise of the central part of each area. This choice of the weight minimizes the final noise value. To improve the signal-to-noise ratio we smoothed the data to a resolution of $1.5^{\prime}$ in both coordinates. Finally, we have computed the resulting noise amplitude for the whole combined field (see Fig. 3). 

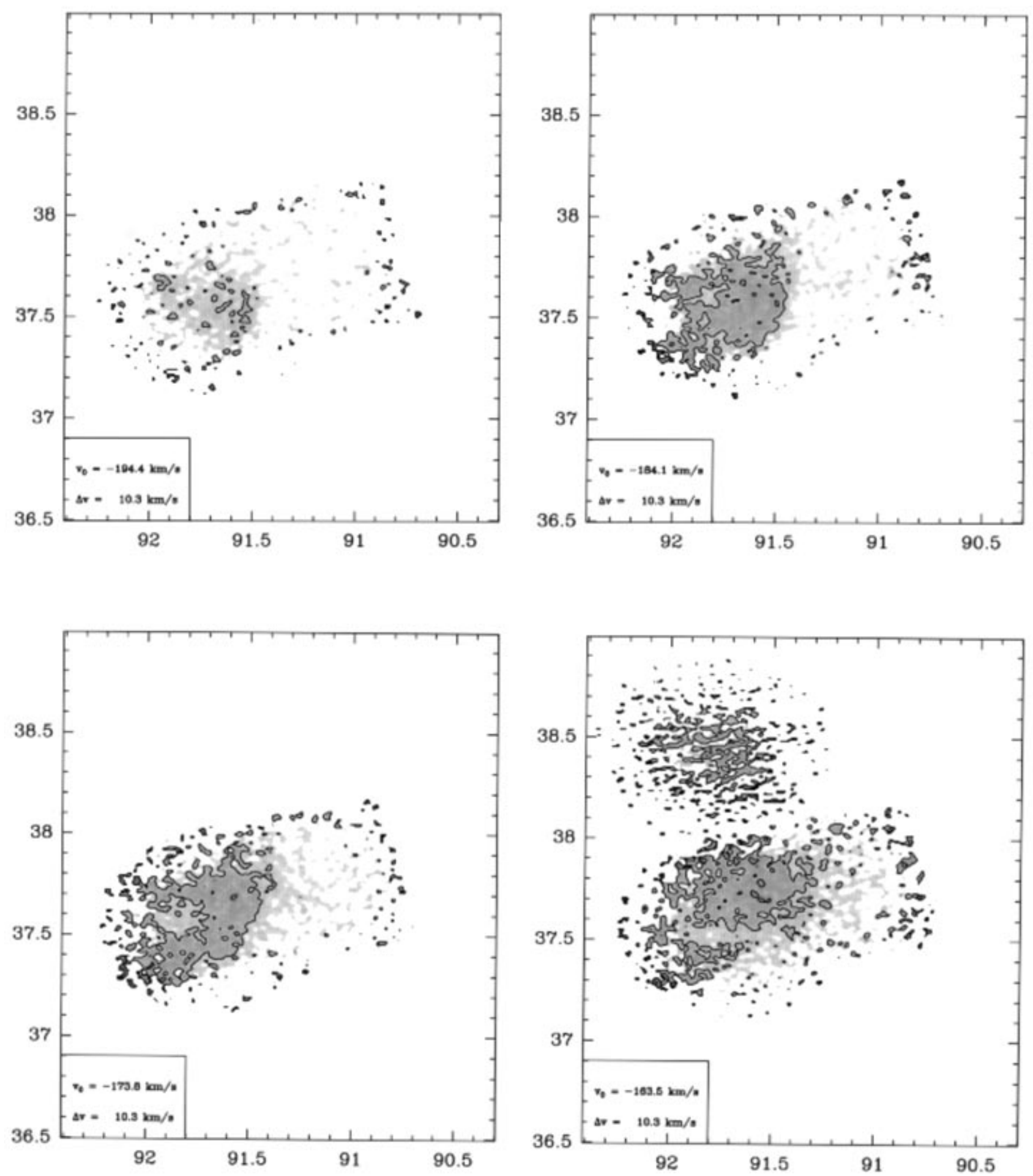

Fig. 4. Channel maps of the mean brightness temperatures for the combined interferometer fields at the edge of the Draco nebula. The central velocities as well as the velocity ranges are indicated. The grey scale range from white to black corresponds to mean brightness temperatures from 0 to $7 \mathrm{~K}$ at $-194.4<v<-116.6 \mathrm{~km} \mathrm{~s}^{-1}$, from 0 to $5 \mathrm{~K}$ at $-50.2<v<34.7 \mathrm{kms} \mathrm{s}^{-1}$ and from 1.5 to $20 \mathrm{~K}$ at $-30.9<v<-7.7 \mathrm{~km} \mathrm{~s}^{-1}$. The contours are at 0.75 and $1.5 \mathrm{~K}$ at $-194.4<v<-116.6 \mathrm{~km} \mathrm{~s}, 2,3,4$ and $5 \mathrm{~K}$ at $-50.2<v<34.7 \mathrm{~km} \mathrm{~s}^{-1}$ and $4,6.5,9.5,13$ and $17 \mathrm{~K}$ at $-30.9<v<-7.7 \mathrm{~km} \mathrm{~s}^{-1}$ 

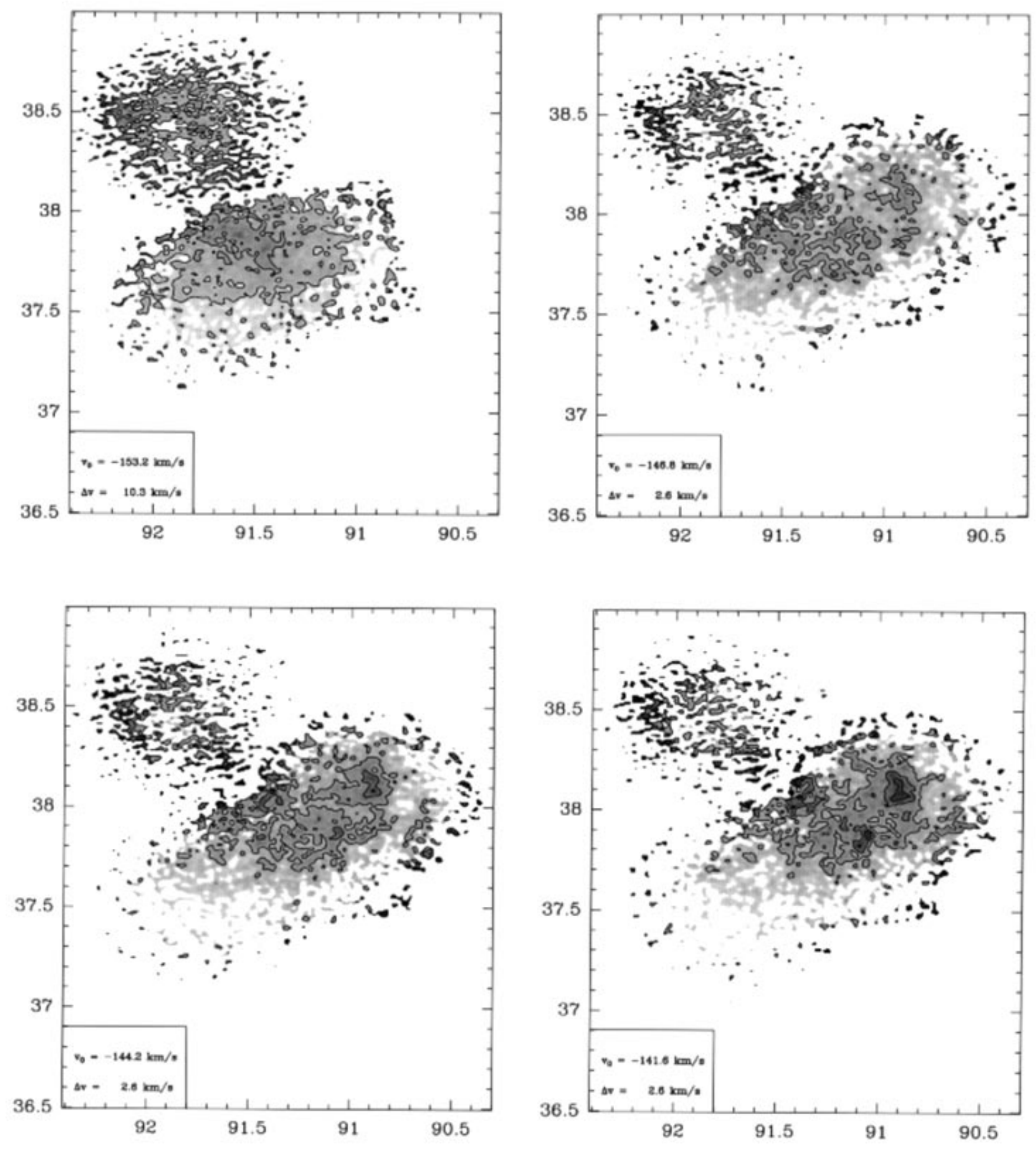

Fig. 4. continued 

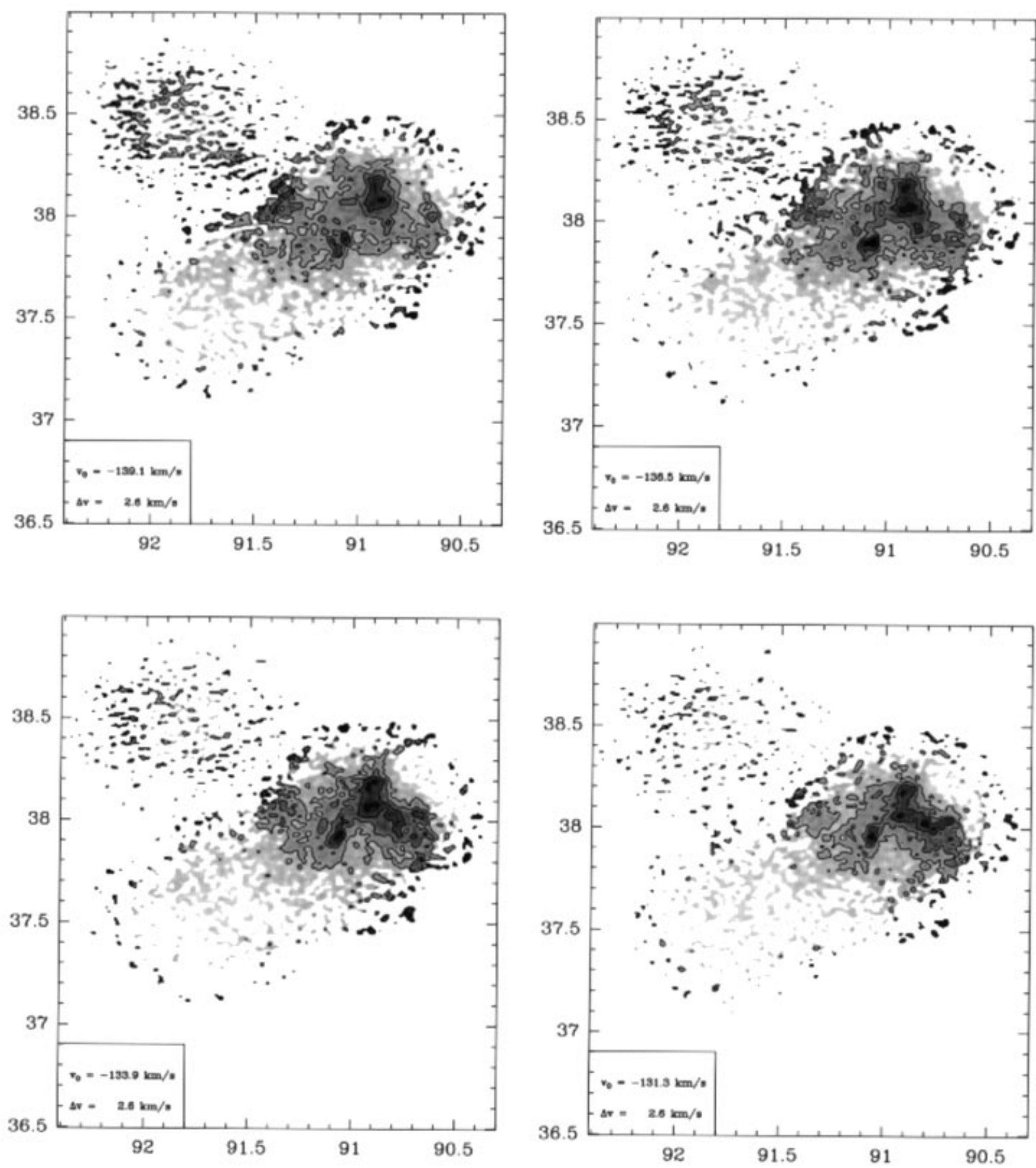

Fig. 4. continued 

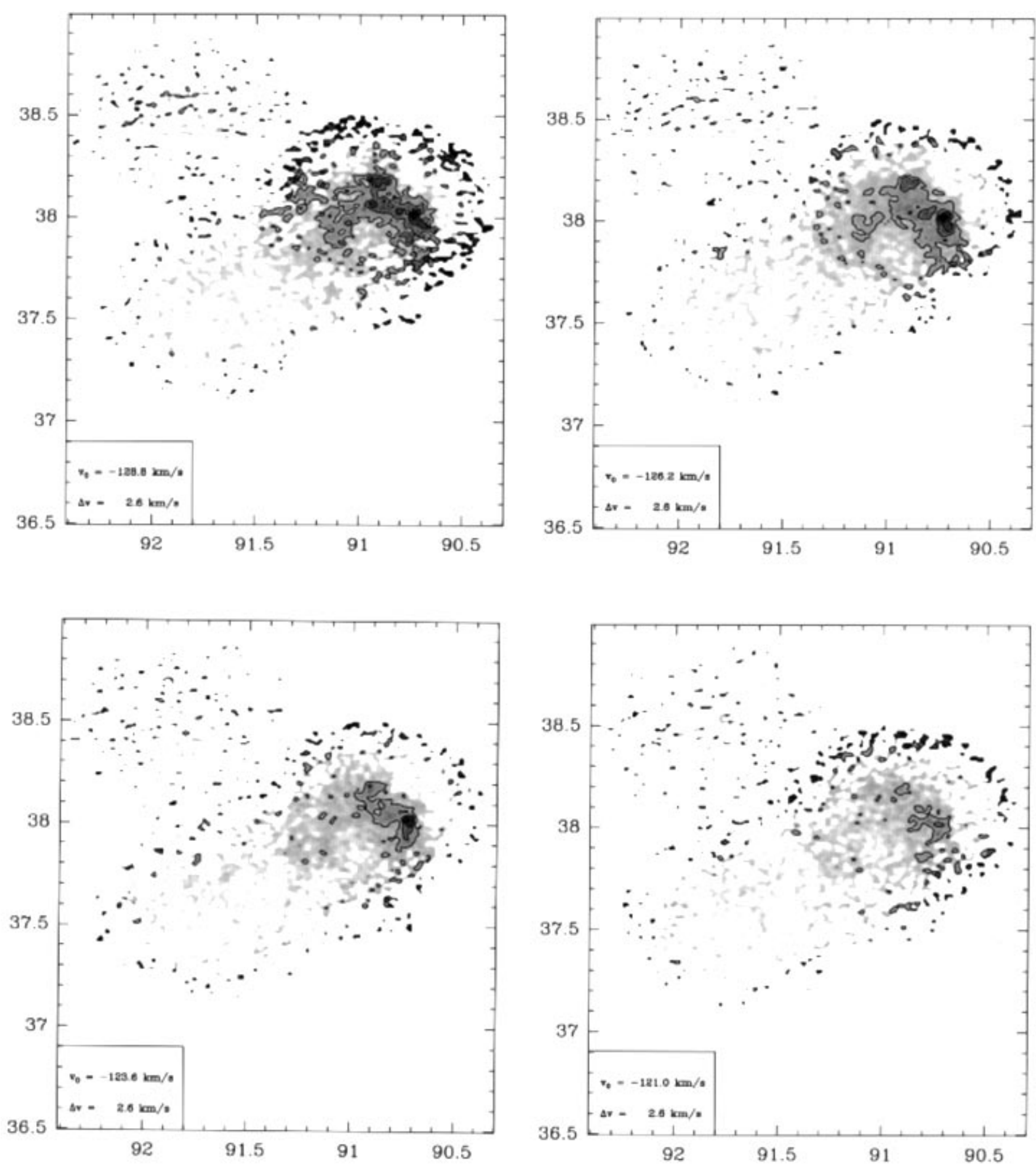

Fig. 4. continued 

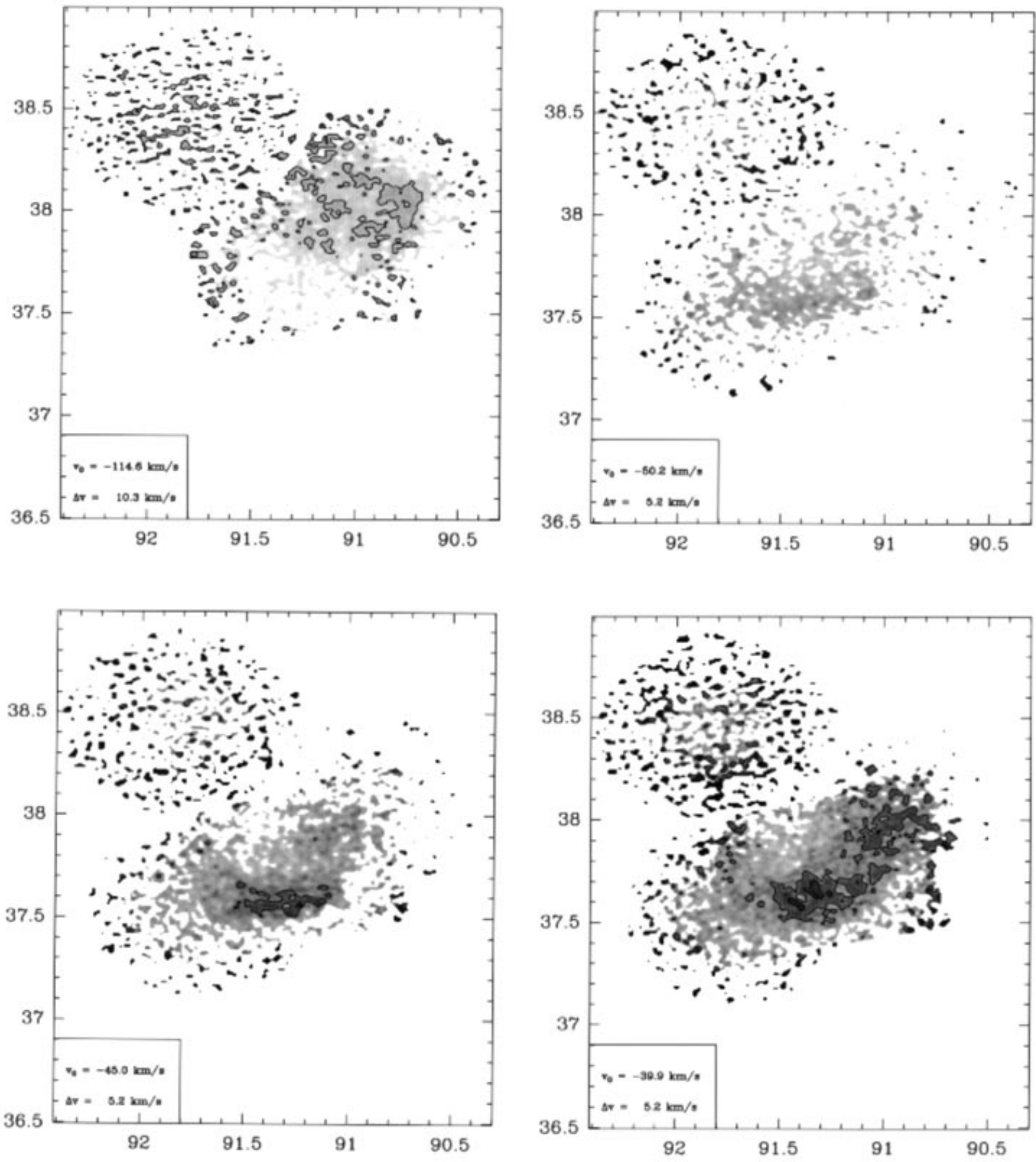

Fig. 4. continued 

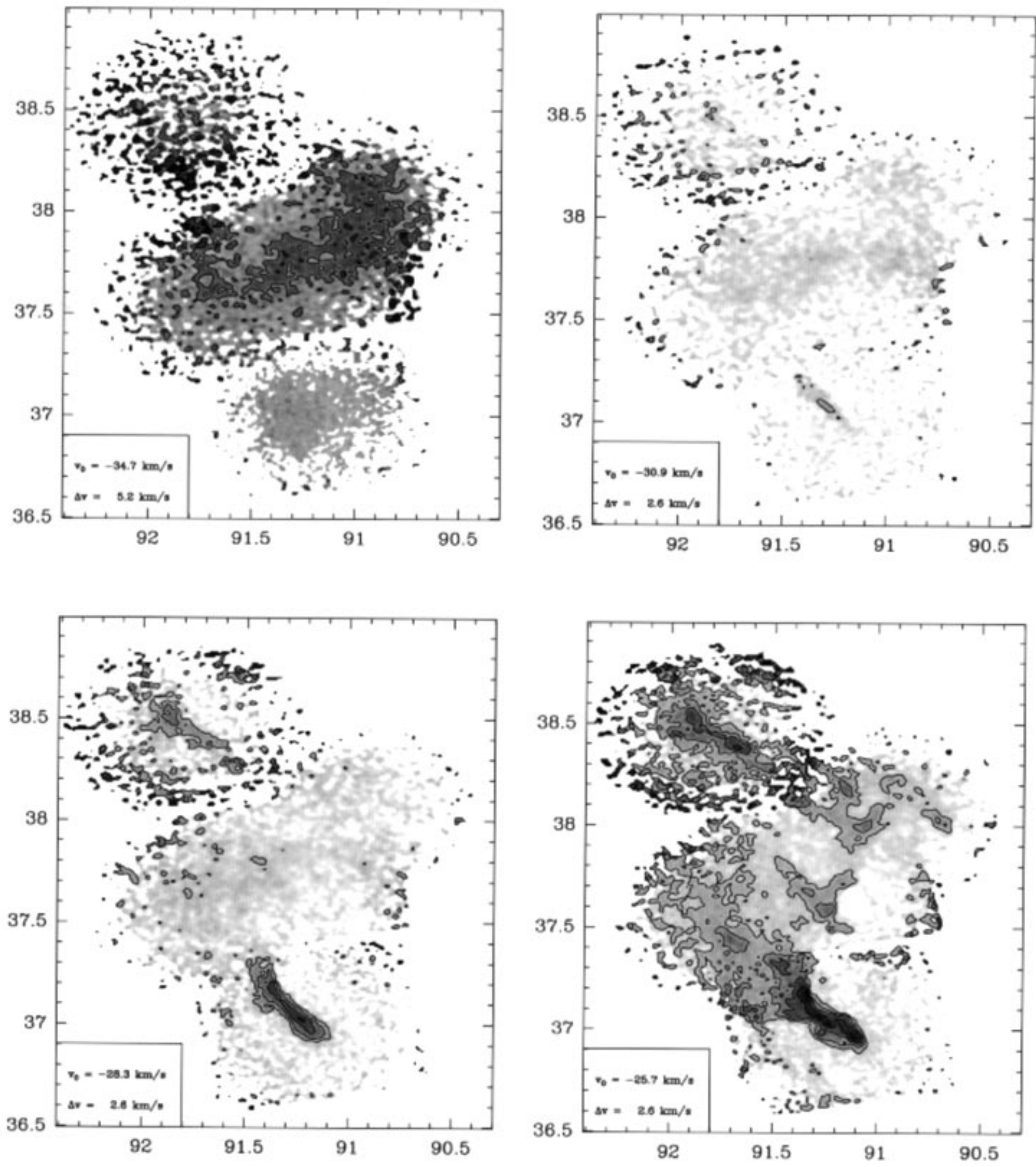

Fig. 4. continued 

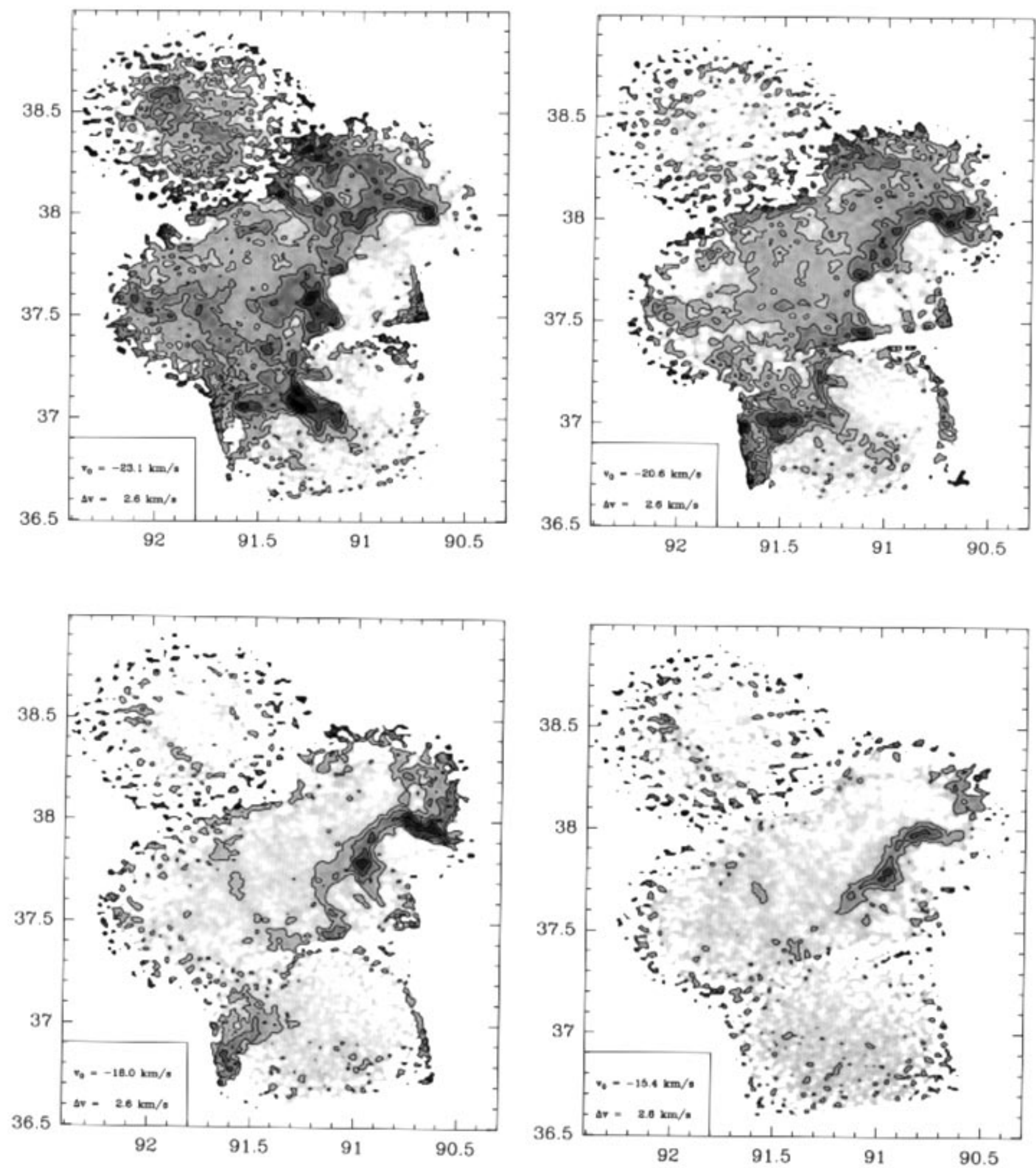

Fig. 4. continued 

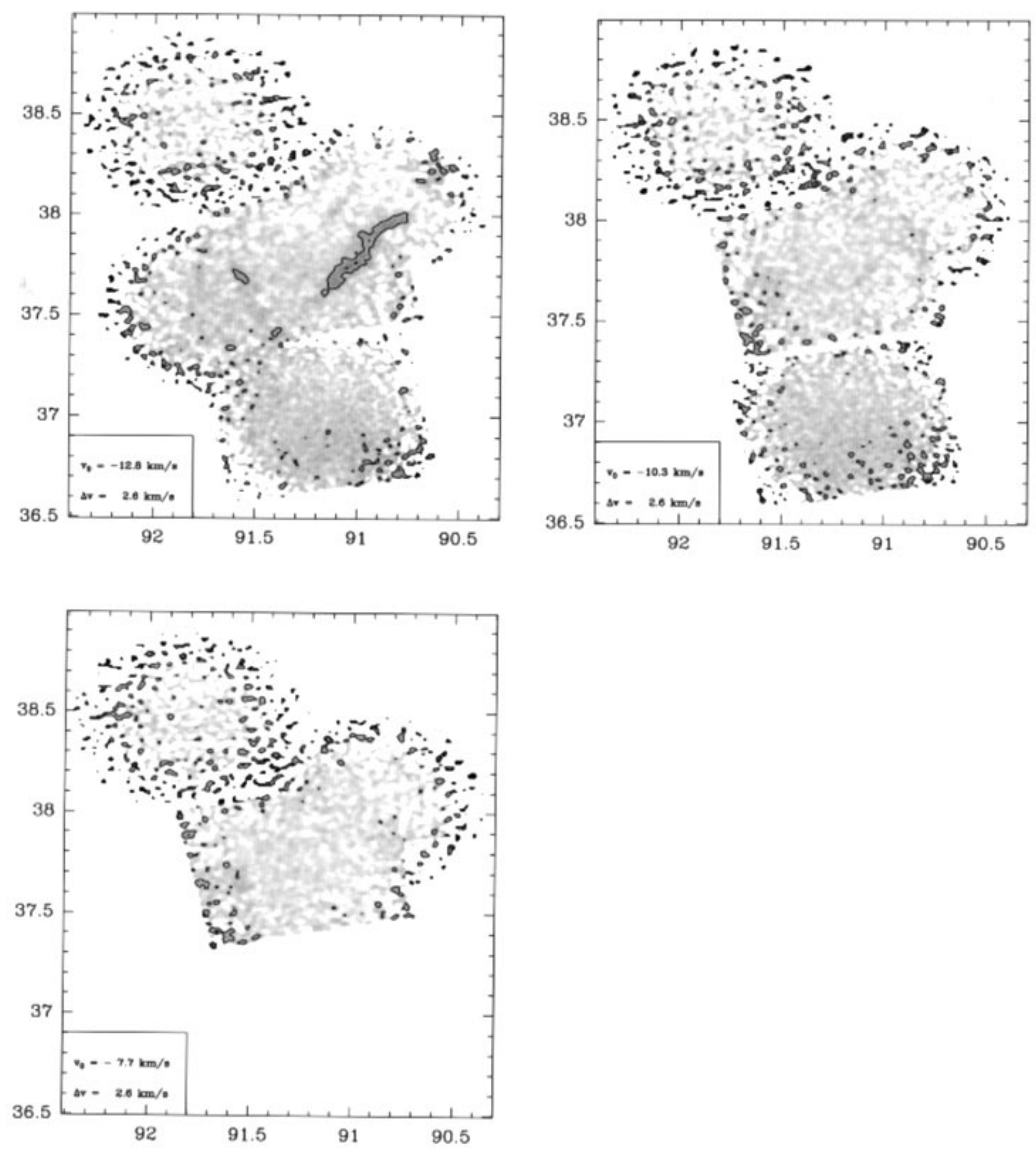

Fig. 4. continued 
Table 6. The observational parameters of the Effelsberg short spacing observations

\begin{tabular}{lclllr}
\hline Field & Date & $\mathrm{RX}$ & $\begin{array}{l}\Delta v \\
\left(\mathrm{~km} \mathrm{~s}^{-1}\right)\end{array}$ & $\begin{array}{l}\delta v \\
\left(\mathrm{~km} \mathrm{~s}^{-1}\right)\end{array}$ & $\begin{array}{c}t_{\text {int }} \\
(\mathrm{s})\end{array}$ \\
\hline WSRT 1 & 1983 & 1 & $(-103,61)$ & 0.32 & 30 \\
& & 2 & $(-103,61)$ & 0.32 & 30 \\
WSRT 2 & \multirow{2}{*}{1984} & 1 & $(-431,230)$ & 1.29 & 112 \\
& & 2 & $(-263,63)$ & 0.64 & 112 \\
VLA 1 & \multirow{2}{*}{1983} & 1 & $(-431,230)$ & 1.29 & 112 \\
& & 2 & $(-431,230)$ & 1.29 & 112 \\
VLA 2 & \multirow{2}{*}{1984} & 1 & $(-431,230)$ & 1.29 & 112 \\
& & 2 & $(-431,230)$ & 1.29 & 112 \\
VLA 3 & \multirow{2}{*}{1988} & 1 & $(-431,230)$ & 1.29 & 48 \\
& & 2 & $(-431,230)$ & 1.29 & 48 \\
VLA 4 & \multirow{2}{*}{1986} & 1 & $(-431,230)$ & 1.29 & 42 \\
& & 2 & $(-431,230)$ & 1.29 & 42 \\
\hline
\end{tabular}
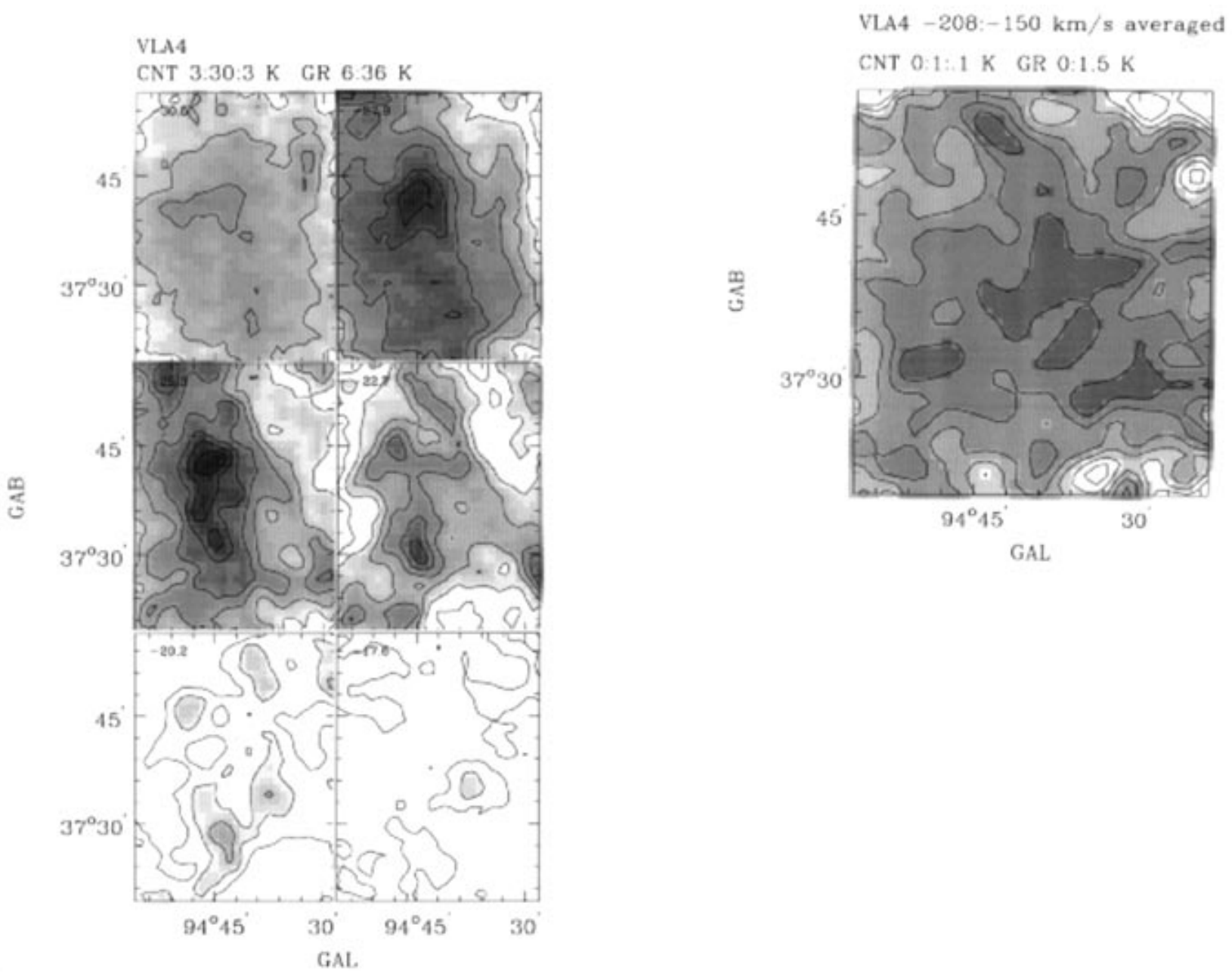

Fig. 5. Channel maps of brightness temperatures for the VLA 4 field. The central velocities as well as the velocity ranges are indicated. Avergaged emission between velocity -208 and $-150 \mathrm{~km} \mathrm{~s}^{-1}$ : The grey scale range from white to black corresponds to brightness temperatures from $0 \mathrm{~K}$ to $1.5 \mathrm{~K}$. The contours start at $T_{\mathrm{B}}=0.0 \mathrm{~K}$. The contour interval is $0.1 \mathrm{~K}$. Emission at velocities from -30.5 to $-17.6 \mathrm{~km} \mathrm{~s}^{-1}$ : The grey scale range from white to black corresponds to brightness temperatures from $6 \mathrm{~K}$ to $36 \mathrm{~K}$. The contours start at $T_{\mathrm{B}}=3.0 \mathrm{~K}$. The contour interval is $3 \mathrm{~K}$ 
Table 7. Main beam solid angle MB of the synthesized beams, conversion factors and noise temperature of the short spacing corrected maps

\begin{tabular}{llllll}
\hline Field names & & $\begin{array}{l}\text { MB } \\
(\text { ster })\end{array}$ & $\begin{array}{l}T_{\mathrm{B}} S^{-1} \\
\left(\mathrm{~K}(\mathrm{~m} J \mathrm{~J} / \text { beam })^{-1}\right)\end{array}$ & $\begin{array}{l}\Delta T \\
(\mathrm{~K})\end{array}$ & $\begin{array}{l}\delta v \\
\mathrm{~km} \mathrm{~s}^{-1}\end{array}$ \\
\hline WSRT 1 & & $5.4210^{-8}$ & 0.29 & 2.0 & 0.52 \\
WSRT 2 & & $5.3310^{-8}$ & 0.30 & 0.8 & 1.03 \\
VLA 1 & HV & $8.5510^{-8}$ & 0.187 & 0.5 & 2.58 \\
& $\mathrm{LV}$ & $9.3310^{-8}$ & 0.171 & 0.8 & 2.58 \\
VLA 2 & 1984 HV & $8.5610^{-8}$ & 0.186 & 0.7 & 2.58 \\
& $1984 \mathrm{LV}$ & $9.8910^{-8}$ & 0.161 & 1.1 & 2.58 \\
VLA 2 & 1985 HV & $8.9910^{-8}$ & 0.178 & 0.7 & 1.29 \\
& $1985 \mathrm{LV}$ & $8.9510^{-8}$ & 0.178 & 1.5 & 1.29 \\
VLA 3 & $\mathrm{HV}$ & $9.1310^{-8}$ & 0.175 & 1.0 & 2.58 \\
& $\mathrm{LV}$ & $1.0910^{-7}$ & 0.147 & 1.2 & 2.58 \\
VLA 4 & $\mathrm{HV}$ & $8.6210^{-8}$ & 0.185 & 0.5 & 2.58 \\
& $\mathrm{LV}$ & $8.5510^{-8}$ & 0.187 & 1.0 & 2.58 \\
\hline
\end{tabular}

Table 8. Parameters of the combination of the two VLA 2 data sets

\begin{tabular}{|c|c|c|c|c|}
\hline $\begin{array}{l}v \text {-Interval } \\
\left(\mathrm{km} \mathrm{s}^{-1}\right)\end{array}$ & $\begin{array}{l}\text { Weight } \\
1984\end{array}$ & $\begin{array}{l}\text { Weigth } \\
1985^{a}\end{array}$ & $\begin{array}{l}\delta v \\
\left(\mathrm{~km} \mathrm{~s}^{-1}\right)\end{array}$ & $\begin{array}{l}\Delta T \\
(\mathrm{~K})\end{array}$ \\
\hline$(-170.0,-149.4)$ & 1 & 0 & 2.576 & 0.7 \\
\hline$(-147.7,-110.3)$ & $2 / 6$ & $5 / 6,-1 / 6$ & 1.288 & 0.6 \\
\hline$(-108.2,-92.7)$ & 1 & 0 & 2.576 & 0.7 \\
\hline$(-90.1,-41.2)$ & 1 & 0 & 2.576 & 1.5 \\
\hline$(-39.4,-12.4)$ & $2 / 6$ & $5 / 6,-1 / 6$ & 1.288 & 1.3 \\
\hline$(-11.1,-0.8)$ & 0 & 1 & 1.288 & 1.5 \\
\hline
\end{tabular}

${ }^{a}$ : For two overlapping channels both weights are given.

\subsection{The high resolution channel maps}

Figure 4 shows the resulting channel maps of the combined fields along the edge of the Draco nebula. When no strong intensity variations between adjacent channel maps were apparent we averaged the corresponding maps together. In the maps of Fig. 4, at velocities -40 to $-15 \mathrm{kms}^{-1}$, the bright structures at $(l, b) \approx\left(90.75^{\circ}, 37.5^{\circ}\right)$ and $\left(90.65^{\circ}, 37.0^{\circ}\right)$ are artefacts which are due to grating rings. Figure 5 shows channel maps of the field VLA 4. In this sample of the central area of the Draco nebula only a few bright small-scale structures are found.

\section{Some results of the interferometer observations}

The fields selected for aperture synthesis observations were choosen to sample areas of the Draco nebula which showed spatially unresolved structures in the Effelsberg data. Most of the fields (WSRT 1 and 2 and VLA 1 and 2) sample the steep HI boundary at the low-l, low-b side of the nebula. This is the boundary for which Goerigk et al. (1983) found indication of a velocity gradient perpendicular to the main direction of this boundary and showing less negative velocities when moving away from the nebula. For comparison purposes we also sampled a bright HI structure (VLA4) locted at the large-l side of the nebula and a field (VLA3) in the central region of the nebula which shows strong HVC emission.

We find that the brightest structures are found in the (low- $l$, low- $b$ ) boundary. This is true for the intermediate negative velocity range (IVC-range) of the Draco nebula $\left(-40<V<-10 \mathrm{kms}^{-1}\right)$ as well as for the high negative velocity range $\left(-200<V<-100 \mathrm{kms}^{-1}\right)$ of the HVCs which both happen to coincide with the (low-l, lowb) boundary of the Draco nebula. Obviously, the brightest emission in the IVC range is located in a narrow ridge along this boundary.

We confirm the velocity gradient seen for the first time by Goerigk et al. (1983) and find that this velocity gradient is rather steep but is resolved. We further find that our data are consistent within the error limits with the preliminary data used by Mebold \& Kalberla (1984) and by Rohlfs et al. (1989) in their discussion of a molecular cloud which is associated with the Draco nebula and with HVCs.

Finally we point out a positional coincidence of a high brightness HVC at $(l, b, v)=90.71,38.01$, $-123.6 \mathrm{kms}^{-1}$ ) with a depression in the HI emission in the IVC range. This depression is even more striking in 
CO $(J=1-0)$ data taken with the Kitt Peak 12-m Radiotelescope (Mebold et al., in prep). This anti-correlation of HI brightness in the HVC and the IVC velocity range supports the arguments for a spatial association of the Draco nebula and some of the HVCs in the area.

A more detailed discussion of the results and an interpretation will be presented in a separate paper.

Acknowledgements. We would like to thank the staff of the WSRT, Effelsberg and the VLA observatories for their suport to this project. K. Begeman was very helpful in installing GIPSY in Bonn. This project was supported by the Deutsche Forschungsgemeinschaft under project Me 745/7-1/2.

\section{References}

Allen R.J., Ekers R.D., Terlouw J.P., 1985, The Groningen Image Processing System. In: Di Gesù V.D., Scarsi L., Crane P., Friedman J.H., Levialdi S. (eds.) Data analysis in Astronomy. Plenum Press, New York, London, p. 271

Bajaja E., van Albada G.D., 1979, A\&A 75, 251

Bos A., Raimond E., van Someren Grèwve H.W., 1981, A\&A 98,251

Burrows D.N., Mendenhall J.A., 1991, Nat 351, 629

Goerigk W., Mebold U., 1986, A\&A 162, 279

Goerigk W., Mebold U., Reif K., Kalberla P.M.W., Velden L., 1983, A\&A 120, 63

Hartmann Dap, 1994, PhD Thesis, Sterrewacht Leiden

Heiles C., 1984, ApJS 55, 585

Heiles C., Reach W.T., Koo B.-C., 1988, ApJ 332, 313

Herbstmeier U., 1984, Diploma Thesis, Universität Bonn

Herbstmeier U., Heithausen A., Mebold U., 1993, A\&A 272 , 514

Herbstmeier U., Kerp J., Moritz P., 1994a, Rev. Mod. Astr., 7,151

Herbstmeier U., Moritz P., Heithausen A., 1994b, Where is the molecular hydrogen in the Draco nebula? In: Cutri R.M., Latter W.B. (eds.), Proc. The First Symposium on the Infrared Cirrus and Diffuse Molecular Clouds, A.S.P. Conf. Ser. 58 , p. 176

Hirth W., Mebold U., Müller P., 1985, A\&A 153, 249

Hirth W., Mebold U., Dahlem M., Müller P., 1991, A\&SS 186, 211

Högbom J.A., Brouw W.N., 1974, A\&A 33, 289

Kalberla P.M.W., Mebold U., Reich W., 1980, A\&A 82, 275

Kalberla P.M.W., Mebold U., Reif K., 1982, A\&A 106, 190

Kalberla P.M.W., Herbstmeier U., Mebold U., 1984, A neutral gas jet in a low velocity shock front at the boundary of the Draco nebula. In: Bruhweiler F.C., Kondo Y., Savage
B.D. (eds.), Proc. IAU Coll. 81, Local Interstellar Medium, NASA CP-2345, p. 243

Kerp J., 1994, A\&A 289, 357

Lada E.A., Blitz L., 1988, ApJ 326, L69

Lilienthal D., Wennmacher A., 1990, A\&A 235, L13

Lilienthal D., Wennmacher A., Herbstmeier U., Mebold U., 1991, A\&A 250, 150

Lynds B.T., 1965, ApJS 12, 163

Magnani L., Blitz L., Mundy L., 1985, ApJ 295, 402

Marshall F.J., Clark G.W., 1984, ApJ 287, 633

McCammon D., Burrows D.N., Sanders W.T., Kraushaar W.L., 1983, ApJ 269, 107

Mebold U., Cernicharo J., Velden L., et al., 1985, A\&A 151, 427

Mebold U., Heithausen A., Reif K., 1987, A\&A 180, 213

Mebold U., Herbstmeier U., Kalberla P.M.W., Souvatzis I., 1989, Molecules at the interface of an HVC and a high-z H I filament. In: Tenorio-Tagle G., Moles M., Melnick J. (eds.), Proc. IAU Coll. 120, Structure and Dynamics of the Interstellar Medium, Lecture Notes in Physics 315. Springer, Berlin, Heidelberg, New York, p. 424

Mebold U., de Boer K.S., Wennmacher A., 1990, High velocity clouds and their interaction with the galactic disk. In: Sanchez F., Vazquez M. (eds.), Proc. XIth European Regional Astronomy Meeting of the IAU, New Windows to the Universe, Vol. II. Cambridge University Press, p. 413

Mebold U., Kerp J., Herbstmeier U., Moritz P. Westphalen G., 1993, Adv. Sp. Res. 13(12), 93

Mebold U., Kalberla, P.M.W., 1984, NASA Conference Publication 2345, Local Interstellar Medium, IAU Colloq. No. 81,248

Minkowski R., Silk J., Siluk R.S., 1972, ApJ 175, L123

Moritz P., 1995, PhD Thesis (in preparation)

Odenwald S.F., 1988, ApJ 325, 320

Odenwald S.F., Rickard L.J., 1987, ApJ 318, 702

Penprase B.E., 1992, ApJS 83, 273

Rohlfs R., 1986, Diploma Thesis, Universität Bonn

Rohlfs R., 1989, PhD Thesis, Universität Bonn

Rohlfs R., Herbstmeier U., Mebold U., Winnberg A., 1989, A\&A 211, 402

Schmitz J., 1989, Diploma Thesis, Universität Bonn

Snowden S.L., Mebold U., Hirth W., Herbstmeier U., Schmitt J.H.M.M., 1991, Sci 252, 1529

Souvatzis I., 1989, Diploma Thesis, Universität Bonn

Trümper J., 1983, Adv. Sp. Res. 2(4), 241

Verschuur G.L., 1990, ApJ 361, 497

Wakker B.P., 1991, A\&A 250, 499

Wennmacher A., 1988, Diploma Thesis, Universität Bonn

Wennmacher A., 1994, PhD Thesis, Universität Bonn 